\title{
Nonperturbative definition of the standard models
}

\author{
Juven Wang $\oplus^{1,2, *}$ and Xiao-Gang Wen ${ }^{3}$ \\ ${ }^{1}$ School of Natural Sciences, Institute for Advanced Study, Princeton, New Jersey 08540, USA \\ ${ }^{2}$ Center of Mathematical Sciences and Applications, Harvard University, Cambridge, Massachusetts 02138, USA \\ ${ }^{3}$ Department of Physics, Massachusetts Institute of Technology, Cambridge, Massachusetts 02139, USA
}

(Received 14 June 2019; accepted 21 May 2020; published 17 June 2020)

\begin{abstract}
The standard models contain chiral fermions coupled to gauge theories. It has been a longstanding problem to give such gauged chiral fermion theories a quantum nonperturbative definition. By classification of quantum anomalies (including perturbative local anomalies and nonperturbative global anomalies) and symmetric interacting invertible topological orders via a mathematical cobordism theorem for differentiable and triangulable manifolds, and by the existence of a symmetric gapped boundary (designed for the mirror sector) on the trivial symmetric invertible topological orders, we propose that Spin(10) chiral fermion theories with Weyl fermions in 16-dimensional spinor representations can be defined on a $3+1 \mathrm{D}$ lattice without fermion doubling, and subsequently dynamically gauged to be a Spin(10) chiral gauge theory. As a result, the standard models from the $16 n$-chiral fermion $\mathrm{SO}(10)$ grand unification can be defined nonperturbatively via a $3+1 \mathrm{D}$ local lattice model of bosons or qubits. Furthermore, we propose that standard models from the 15n-chiral fermion SU(5) Grand Unification can be regularized by a $3+1 \mathrm{D}$ local lattice model of fermions.
\end{abstract}

DOI: 10.1103/PhysRevResearch.2.023356

\section{INTRODUCTION AND DEFINITIONS}

The standard models [1-3], gauge theories with the Lie algebra $u(1) \times s u(2) \times s u(3)$ in $3+1 \mathrm{D}$, coupled to fermions and bosons, are believed to describe elementary particles. ${ }^{1}$ In the standard standard model, there are 15 two-component complex Weyl fermions per family. The SU(5) grand unification [5] has 15 complex Weyl fermions per family. There are also nonstandard standard models, such as the one from the $\mathrm{SO}(10)$ grand unification [6] which has 16 complex Weyl fermions per family. But for a long time, the standard models were only defined via a perturbative expansion, which is known not to converge. So the standard models were not yet known to be well-defined quantum theories. This is related

\footnotetext{
${ }^{1}$ Elementary particles include fermions from quarks and leptons, and bosons from gauge mediators and Higgs particle. Gravitons are not yet discovered experimentally. In addition, in our work, we do not consider any dynamical gravity; we only consider anomalies of gauge or gravitational nondynamical background fields. The local Lie algebra of standard standard models is $u(1) \times s u(2) \times s u(3)$, but the global structure Lie group can be $\frac{\mathrm{U}(1) \times \mathrm{SU}(2) \times \mathrm{SU}(3)}{\mathbb{Z}_{q}}$, where $q=1,2,3,6$; see a recent overview [4] on this issue. In fact, as we will show later, for $\mathrm{SO}(10)$ and $\mathrm{SU}(5)$ grand unifications, it is more natural to study the case $q=6$. Also, we denote the $d$-dimensional space and one-dimensional time as $d+1 \mathrm{D}$.

*Corresponding author: jw@cmsa.fas.harvard.edu

Published by the American Physical Society under the terms of the Creative Commons Attribution 4.0 International license. Further distribution of this work must maintain attribution to the author(s) and the published article's title, journal citation, and DOI.
}

to the longstanding gauged chiral fermion problem: how to define a chiral fermion theory, with the parity violation [7], coupled to the gauge field, nonperturbatively and in the same dimension, as a well-defined quantum theory with a finitedimensional Hilbert space for a finite-size system (for details, see Appendix A 1), but without suffering from fermion doublings [8]. In this work, we use the term gauged chiral fermion theory to mean chiral fermion theory coupled to a nondynamical background gauge field. In fact, the gauge theories focused on in this article are mostly nondynamical, unless mentioned otherwise.

There were many previous pioneer attempts, such as a lattice gauge approach [9], Ginsparg-Wilson fermion approach [10], domain-wall fermion approach [11,12], and overlapfermion approach $[13,14]$. In the Ginsparg-Wilson fermion approach, the to-be-gauged symmetry is not strictly an on-site symmetry, but only a quasilocal symmetry (see Definition 2 and [15-18]; the quasilocal symmetry is still a non-on-site symmetry), and thus it is very challenging to gauge. (The Abelian chiral gauge theory is achieved by Ref. [14]; however, the non-Abelian case is still an open question). In the domainwall fermion approach, we have an extra dimension, where the dynamical gauge fields can propagate. The overlap-fermion approach is a reformulation of the domain-wall fermion approach. The above approaches normally start with a spacetime Euclidean lattice path integral and implement the GinspargWilson fermion.

In contrast, in our work, we do not formulate a spacetime lattice path integral or Ginsparg-Wilson fermion. Instead, we consider a discretized spatial lattice Hamiltonian with a continuous time, with additional criteria (see Definition 1): (1) with a tensor product Hilbert space, (2) with all interaction terms bounded by a finite range of lattice spacings (called 
short-range interactions), and (3) we only discuss on-site symmetries (see Definition 2). Below we refer to our setup as a local lattice model. ${ }^{2}$

In this work, we aim to show nontrivial evidence that the gauged chiral fermion problem in both the $16 n$-fermion and the $15 n$-fermion standard models can be solved via a generalized lattice gauge approach under local lattice model (Definition 1). In the standard lattice gauge approach, the fermions do not interact directly. The generalized lattice gauge approach simply adds an extra direct fermion interaction or an indirect fermion interaction via some Higgs fields. A generalized lattice gauge approach, called the mirror fermion approach, was proposed in 1986 [19,20]. In such an approach, one starts with a lattice model containing chiral fermions (named the chiral sector or the normal sector) and a chiral conjugated mirror sector (the mirror sector), with a to-be-gauged symmetry acting as an on-site symmetry. Then, one includes a proper fermion interaction [21,22] in such a local lattice model, attempting to gap out the mirror sector completely, without breaking the on-site symmetry and without affecting the low-energy properties of the normal sector. This is the key step, which will be referred to as gapping out the mirror sector without breaking the (to-be-gauged) symmetry. Lastly, one can gauge the on-site symmetry to obtain a gauged chiral fermion theory, regularized by a local lattice model. ${ }^{3}$

Reference [19] proposed a way to gap out the mirror sector without breaking the symmetry, by introducing composite fermion fields formed by mirror fermion fields, and by adding symmetric mass terms between composite fermion fields and the mirror fermion fields to make all those fermion fields formally massive. However, such a proposal cannot work in general. Even we can make all the fermion fields formally massive; it does not imply we can fully gap out the mirror sector. This is because, even for some models with a perturbative local anomaly, ${ }^{4}$ one can find composite fermion fields formed by mirror fermion fields and to make all those fermion fields formally massive (see the Appendix of Ref. [23], arXiv version). Also, the extensive studies of the previous mirror fermion proposal [24-27] had difficulties to demonstrate that interactions can fully gap out the mirror sector without breaking the symmetry and without modifying the low-energy dynamics of the normal sector. It was pointed out in Ref. [28] that "attempts to decouple lattice fermion doubles by the method of Swift and Smit cannot succeed." Consequently, many people gave up the mirror fermion approach.

\footnotetext{
${ }^{2}$ For a concrete lattice model, we mostly focus on a spatial lattice Hamiltonian. However, our arguments and Propositions 1, 2, and 3 are more general than a Hamiltonian picture; they are also applicable to quantum field theory and spacetime path integral approaches.

${ }^{3}$ Colloquially, we refer to a lattice regularization the same as a lattice realization. When we say a field theory can be regularized (on the boundary of a lattice in one higher dimension), we also mean a field theory can be realized.

${ }^{4} \mathrm{We}$ overview the concepts of anomalies, including perturbative local anomaly and nonperturbative global anomaly in Appendix A. In the main text, however, we purposefully reduce the information on anomalies, but focus on the mathematically very well-defined concepts called the cobordism theory.
}

Recently, Ref. [23] conjectured a different gapping condition for the mirror sector:

Proposition $i$. Consider a mirror sector in $d+1 \mathrm{D}$ with a symmetry group $G$. The mirror sector can be gapped out without breaking the symmetry $G$ if (1) there exist (possibly $G$-symmetry breaking) mass terms that make all the fermions massive, and (2) $\pi_{n}\left(G / G_{\text {grnd }}\right)=0$ for $n \leqslant d+2$, where $G_{\text {grnd }}$ is the unbroken symmetry group.

The above Ref. [23]'s claim is obtained based on the following assumption (not rigorously proven so far):

Proposition ii. A $d+1 \mathrm{D} G$-symmetric nonlinear $\sigma$ model with topologically trivial target space $M=G / G_{\text {grnd }}$ [i.e., $\pi_{n}(M)=0$ for $n \leqslant d+2$ ] allows a gapped $G$-symmetric ground state.

Applying the above propositions, Ref. [23] claimed that $3+1 \mathrm{D} \operatorname{Spin}(10)$ chiral fermion theory with Weyl fermions in a 16-dimensional spinor representation can be defined via an interacting local lattice model with a $\operatorname{Spin}(10)$ on-site symmetry which can be gauged. ${ }^{5}$ The 16 -fermion standard model [i.e., $\mathrm{SO}(10)$ grand unification or $\mathrm{SO}(10)$ grand unified theory $\equiv \mathrm{SO}(10)$ (GUT)] can then be obtained from a $3+1 \mathrm{D}$ Spin(10) chiral gauge theory, coupled to Spin(10) chiral Weyl fermions in the 16-dimensional representation of Spin(10).

Purpose of our present work. The homotopy group argument in Ref. [23] only proposed a sufficient condition. There are mirror sectors (thus also normal sectors) that do not satisfy the condition, but that can still be gapped out without breaking the symmetry and without altering low-energy physics in the normal sector. In this work, we are going to prescribe a more general condition, to capture the cases missed by Ref. [23]:

Proposition 1. Consider a continuum field theory in $d+$ 1D with an internal symmetry group $G_{f} \cdot{ }^{6}$ If the following two conditions hold: (i) if the field theory can be regularized as the low-energy effective theory of a boundary of a gapped local lattice model in one higher dimension $d+2 \mathrm{D}$ with a bulk on-site symmetry $G_{f}$, and (ii) if the gapped ground state of the bulk lattice model represents a trivial cobordism invariant in $d+2 \mathrm{D}$, then, the $d+1 \mathrm{D}$ field theory can be regularized as the low-energy effective theory of a local lattice model in the same dimension $d+1 \mathrm{D}$ with an on-site internal symmetry $G_{f}$.

Our above statement used the following assumption:

Proposition 2. A gapped local lattice model with an on-site internal symmetry $G_{f}$ in $d+2 \mathrm{D}$ must exist a $G_{f}$-symmetric gapped $d+1 \mathrm{D}$ boundary (that does not break the $G_{f}$ symmetry), if its gapped bulk ground state in $d+2 \mathrm{D}$ represents a trivial cobordism invariant in $d+2 \mathrm{D}$.

The existence of a symmetric gapped boundary is based on the belief that the bulk with a trivial cobordism invariant can be smoothly deformed into a symmetric product state without closing the gap. The symmetric product state always have a symmetric gapped boundary. See Appendices A and D for further details.

\footnotetext{
${ }^{5}$ In this work, a local lattice model is a lattice model of bosons and/or fermions with short-range interactions and a tensor-product structured Hilbert space; see Definition 1.

${ }^{6}$ An internal symmetry may or may not be an on-site symmetry. But an on-site symmetry must be an internal symmetry.
} 
To obtain Proposition 1, we have to apply Proposition 2. We first regularize the field theory as a boundary (also referred as the normal sector) of the gapped lattice model in one higher dimension, then assume the lattice model has a finite thickness, and make the boundary on the other side (also referred as the mirror sector) to be the symmetric gapped boundary ensured by Proposition 2.

Using the above statements, we will show that a $3+1 \mathrm{D}$ Spin(10) chiral fermion theory with Weyl fermions in a 16-dimensional spinor representation can be defined via an interacting local lattice model with a Spin(10) on-site internal symmetry which can be gauged. In addition, we will show that a $3+1 \mathrm{D} \mathrm{SU}(5)$ chiral fermion theory with Weyl fermions in five-dimensional and 10-dimensional representations can be defined via an interacting local lattice model with an SU(5) on-site symmetry which can be gauged.

Lastly, we remark that to fully characterize the global symmetry in a fermion system, we need to specify the full internal global symmetry group $G_{f}$ and how the fermion number parity $\mathbb{Z}_{2}^{f}$ is embedded in $G_{f}$. So we can denote the fermion symmetry as $G_{f} \supset \mathbb{Z}_{2}^{f}$. In our case, the full internal symmetry is actually $G_{f}=\operatorname{Spin}(10)$, while $\mathrm{SO}(10)$ is the quotient group $\operatorname{Spin}(10) / \mathbb{Z}_{2}^{f}=\mathrm{SO}(10)$. So, in this work, we use the following name: a $\operatorname{Spin}(10)$ chiral fermion model [rather than an $\mathrm{SO}(10)$ chiral fermion model which was sometimes used by others].

\section{COBORDISM THEORY AND SYMMETRIC GAPPED BOUNDARY}

Let us first explain the cobordism theory used in Propositions 1 and 2. Based on a theorem of Freed-Hopkin [29] and an extended generalization [30-32] (including higher symmetries [33-40]), there is a one-to-one correspondence between "the deformation class of invertible topological quantum field theories $\left(\mathrm{iTQFTs}^{7}\right.$ ) $[41,42]$ with symmetry (including higher symmetries)" and "a cobordism group." More precisely, there

${ }^{7}$ It is called an invertible TQFT because its partition function $\mathbf{Z}\left(M^{D}\right)$ on any closed manifold $M^{D}$ must have its absolute value $\left|\mathbf{Z}\left(M^{D}\right)\right|=1$, namely, $\mathbf{Z}\left(M^{D}\right)=e^{i \theta}$ can only be a complex phase. On a closed spatial manifold $M^{D-1}$, it always has a single ground state $\mathbf{Z}\left(M^{D-1} \times S^{1}\right)=1$ with no topological ground-state degeneracy. Thus, $\mathbf{Z}(M)=e^{i \theta}$ has an inverted phase $\mathbf{Z}^{\dagger}(M)=e^{-i \theta}$ that can be defined as its complex conjugated iTQFT. The combined iTQFT $\mathbf{Z}(M) \cdot \mathbf{Z}^{\dagger}(M)=1$ is the trivial iTQFT (i.e., the trivial gapped vacuum).

${ }^{8}$ By all symmetric iTQFTs, their classifications and characterizations depend on the category of manifolds that can detect them. The categories of manifolds can be TOP (topological manifolds), PL (piecewise linear manifolds), or DIFF (differentiable thus equivalently smooth manifolds), etc. These categories are different and they are related by the following inclusions:

$$
\text { TOP } \supseteq \text { PL } \supseteq \text { DIFF. }
$$

In contrast, triangulable manifolds are smooth manifolds at least for dimensions up to $D=4$ (i.e., the "if and only if" statement is true below $D \leqslant 4$ ). The concept of piecewise linear (PL) and smooth DIFF structures are equivalent in dimensions $D \leqslant 6$. Thus all is a one-to-one correspondence (isomorphism "@”) between the following two well-defined "mathematical objects" (these "objects" turn out to form the Abelian group structures):

$$
\begin{aligned}
& \left\{\begin{array}{c}
\text { Deformation classes of the reflection positive } \\
D \text {-dimensional extended invertible } \\
\text { topological field theories (iTQFTs) with } \\
\text { symmetry group } G=\frac{G_{\text {spacetime }} \times G_{f}}{N_{\text {shared }}}
\end{array}\right\} \\
& \cong\left[M T(G), \Sigma^{D+1} I \mathbb{Z}\right]_{\text {tors }} .
\end{aligned}
$$

The $M T(G)$ is the Madsen-Tillmann spectrum [43] of the $G$ group, the $\Sigma$ is the suspension, the $I \mathbb{Z}$ is the Anderson dual spectrum, and $\Sigma^{D+1} I \mathbb{Z}$ is the $D+1$-th suspension of the spectrum. The tors means taking only the finite group sector (i.e., the torsion group). The right-hand side is the torsion subgroup of the homotopy classes of maps from a Thom-Madsen-Tillmann spectrum $[43,44]$ to a shift of the Anderson dual to the sphere spectrum. The spacetime symmetry $G_{\text {spacetime }}$ and the internal symmetry $G_{f}$, mod out the shared common normal subgroup $N_{\text {shared }}$, is combined to a $G$ structure:

$$
G=\frac{G_{\text {spacetime }} \times G_{f}}{N_{\text {shared }}} .
$$

This also means the pertinent iTQFTs of (2) are defined on manifolds with $G$ structure.

In condensed-matter physics, this roughly means the following:

Proposition 3. There is a one-to-one correspondence [29] between "the invertible gapped states with an internal symmetry $G_{f}$ (including higher symmetries [30,33])" that can be regularized on a lattice with $G_{f}$ realized as an on-site symmetry [45] in its own dimensions and "the group elements as the corresponding generators in a cobordism group for the internal symmetry $G_{f}$," at least in lower dimensions. ${ }^{9}$

symmetric iTQFT classified by the cobordant properties of smooth manifolds have a triangulation (thus a lattice regularization) on a simplicial complex [thus an ultraviolet (UV) competition on a lattice]. This implies a correspondence between "the symmetric iTQFTs (on smooth manifolds)" and "the symmetric invertible topological orders (on triangulable manifolds)" for $D \leqslant 4$. This leads to our application of this mathematical fact on the lattice regularization of symmetric iTQFTs and symmetric invertible topological orders for various standard models of particle physics. In this work, we only focus on the smooth differentiable (DIFF) manifolds and their associated all possible iTQFTs. The tools we use in either case would be a certain version of cobordism theory suitable for a specific category of manifolds.

${ }^{9}$ We clarify that before gauging, the $G_{f}$ symmetry discussed in our setup must be an on-site internal symmetry of the lattice model (see Appendix A). Certainly, this does not exclude the possibility that the lattice model may have a larger symmetry. We stress that the $G_{f}$ in the cobordism calculations is the on-site internal symmetry for

the $G_{f}$-symmetric deformation class of the Hamiltonians.

Thus, we consider many $G_{f}$-symmetric Hamiltonians under the $G_{f^{-}}$symmetric preserving deformations. The on-site $G_{f}$ always needs to be preserved in order to be gauged later. For example, we choose 
There is a logic gap here to establish Proposition 3 since, by (2), we only know that there is a one-to-one correspondence between "the iTQFTs with symmetry" and "the cobordism invariants from a cobordism group." We do not yet know if there is a one-to-one correspondence between "the lattice invertible topological order with symmetry" and "the iTQFTs with symmetry." In particular, we do not mathematically and rigorously prove how to construct a lattice Hamiltonian realization for each iTQFT with symmetry classified by a cobordism group. (We remark that some of the "lattice invertible topological orders with symmetry on a lattice" are also called the symmetry-protected topological/trivial states (SPTs) $[15,16,46,47]$, if they can deform to a trivial tensor product state under local unitary transformations after explicitly breaking the symmetry). Regardless of a logic gap in the rigorous mathematical sense, the broad literature suggests strong physical evidence of the following:

(a) The classification of iTQFT $[29,30,48-50]$ so far matches with the classification of lattice invertible topological orders and lattice SPTs [47,51-53]. Many such iTQFTs can thus be constructed on the lattice Hamiltonian.

(b) Moreover, in (2), we only focus on iTQFTs definable on differentiable and triangulable manifolds, and thus those iTQFTs may be regularized by the same lattice from the simplicial complex of triangulable manifolds.

In summary, based on the support of (a) and (b), below we propose and assume that a refined and rigorous version of Proposition 3 is true.

Proposition 2 can be obtained from Proposition 3. There can be two kinds of gapped fermion systems on a lattice: those with topological excitations (which may be fractionalized) and those without topological excitations (i.e., all the excitations correspond to the original fermions or bosons). By definition, the gapped states with topological excitations are the lattice noninvertible topological orders. The gapped states without topological excitations are the "lattice invertible topological orders with symmetry." According to Proposition 3 , if a "lattice invertible topological order with symmetry" has a trivial cobordism invariant, then it must be a "trivial lattice

an internal symmetry group $G_{f}=\operatorname{Spin}(10)$ for SO(10) GUTs. However, we point out that the full symmetry group $G$ used in cobordism calculations also includes the emergent spacetime symmetry at lowenergy infrared (IR) as $G_{\text {spacetime }}=\operatorname{Spin}(D)$ (for a $D$-dimensional Euclidean spacetime). Thus, for the $\operatorname{Spin}(10)$ fermion model, in Eq. (3), we have $N=\mathbb{Z}_{2}^{f}$, so $G=\left(\frac{(\operatorname{Spin}(D) \times \operatorname{Spin}(10))}{\mathbb{Z}_{2}^{f}}\right)$. Given a lattice model, there can be a larger symmetry $G_{\text {onsite }} \supset G_{f}$. Such as some lattice models in Sec. III and in Appendix B, we have

$$
G_{\text {onsite }} \supset \mathrm{U}\left(16 N_{f}\right) \supset \mathrm{U}(16) \supset G_{f}=\operatorname{Spin}(10),
$$

for some flavor number $N_{f}$. There are also other space-group symmetries on a lattice, say $G_{\text {lattice,space, while }} G_{\text {lattice,space }}$ is typically smaller than the emergent $G_{\text {spacetime }}$, so usually

$$
G_{\text {lattice,space }} \subset G_{\text {spacetime }} \text {. }
$$

Overall, all these "symmetries" are not crucial to our discussion; the only key symmetries are the on-site internal $G_{f}$ symmetry, and the overall $G$. invertible topological order with symmetry." ${ }^{10}$ In other words, there exists a symmetry-preserving local unitary transformation that deforms the "trivial lattice invertible topological order with symmetry" into a "trivial tensor product state with symmetry" [54], where its gapped symmetric boundary can always be constructed. (We provide more steps along with these logical arguments in Appendix A 2). Crucially, this is precisely why the cobordism approach allows us to obtain the gapping condition for the mirror sector.

Proposition 1 can be obtained from Proposition 2, if we can show that the normal sector or the mirror sector can be regularized as some boundary states of a gapped local lattice model. We will provide such a local lattice model construction for the Spin(10) chiral fermion theory in Sec. III and in Appendix B as an example.

Although we propose Propositions 1, 2, and 3, we do not require the complete versions of all these propositions to establish our claim of a local lattice model with a chiral fermion low-energy spectrum. We only require the weaker Proposition 1. Let us clarify:

(i) Proposition 1's "the existence of a fully gapped boundary" is a static statement. On the other hand, "the gapless sector can be fully gapped out without breaking the symmetry" is a dynamic statement, more challenging than Proposition 1. But the two statements are related; their detailed relations are given in Appendices A 2 and D, based on physical intuitions of phase boundaries and quantum phase transitions. In fact, we only require the weaker static statement in Proposition 1 's "the existence of a fully gapped boundary" in order to establish the gapped mirror sector.

(ii) To use Proposition 3, we only require a local lattice construction for the cobordism class whose boundary gives rise to the normal sector or mirror sector. To establish Spin(10), Spin(18), and SU(5) chiral fermion theories, we only require a local lattice construction of the trivial cobordism class (the identity element 0 in the cobordism group). They happen to be a trivial bulk gapped insulator in which we certainly can construct their local lattice model with a gapless normal sector on the boundary (Sec. III).

In the following sections, we also provide the physics interpretations of the classifications of all $4+1 \mathrm{D}$ iTQFTs whose boundaries are associated with the $3+1 \mathrm{D} \operatorname{Spin}(10)$ and $\operatorname{Spin}(18)$ chiral fermion theories [for $\mathrm{SO}(10)$ and $\mathrm{SO}(18)$ GUTs] in Sec. III, ${ }^{11}$ and the $3+1 \mathrm{D}$ SU(5) chiral fermion theories [for $\mathrm{SU}(5) \mathrm{GUTs}$ ] in Sec. IV. We relegate the

\footnotetext{
${ }^{10}$ A "trivial lattice invertible topological order with symmetry" means the "trivial gapped vacuum with symmetry" in quantum field theory, or the "symmetric gapped direct product state" in condensed matter.

${ }^{11}$ To be precise, in order to embed the standard standard-model-like spacetime-and-internal symmetry group with this $G=\frac{G_{\text {spacetime }} \times G_{f}}{N_{\text {shared }}}$ structure (3) to the $\mathrm{SO}(10)$ grand unification's $\frac{\operatorname{Spin}(D) \times \operatorname{Spin}(10)}{\mathbb{Z}_{2}^{f}}$, it is natural to consider an alternative standard standard model spacetime-and-internal group $\operatorname{Spin}(D) \times \frac{\mathrm{U}(1) \times \mathrm{SU}(2) \times \mathrm{SU}(3)}{\mathbb{Z}}$, while their gauge Lie algebra is still $u(1) \times s u(2) \times s u(3)$. Here, $\operatorname{Spin}(10)$ and $\frac{\mathrm{U}(1) \times \mathrm{SU}(2) \times \mathrm{SU}(3)}{\mathbb{Z}_{6}}$ are their gauge groups, respectively. See more discussions in footnote 18 .
} 
mathematical calculation details on algebraic topology in Appendix E. (See, also, Ref. [55]).

\section{Spin $(N)$ CHIRAL FERMION THEORY, AND SO(10) AND SO(18) GRAND UNIFICATION}

We now construct a local lattice Hamiltonian model. A $3+$ 1D two-component Weyl fermion described by a Hamiltonian (Model 1 defined in Appendix A 1),

$$
H=\psi^{\dagger} \mathrm{i} \sigma^{i} \partial_{i} \psi, \quad \sigma^{1,2,3} \text { are Pauli matrices, }
$$

can be regularized on the boundary of a fermion hopping model on a 4D spatial cubic lattice with a Hamiltonian operator [23],

$$
\hat{H}_{\text {hop }}=\sum_{i j}\left(t_{i j}^{a b} \hat{c}_{a, i}^{\dagger} \hat{c}_{b, j}+\text { H.c. }\right),
$$

which has four fermion orbitals $(a, b=1, \ldots, 4)$ per site $(\boldsymbol{i}, \boldsymbol{j}$ for sites). The H.c. contains the Hermitian conjugate term. The $4 \times 4$ hopping matrices $t_{i j}$ are given by

$$
\begin{aligned}
& H_{4 \mathrm{D}}\left(k_{1}, k_{2}, k_{3}, k_{4}\right) \\
& =2\left[\Gamma^{1} \sin \left(k_{1}\right)+\Gamma^{2} \sin \left(k_{2}\right)+\Gamma^{3} \sin \left(k_{3}\right)+\Gamma^{4} \sin \left(k_{4}\right)\right] \\
& \quad+2 \Gamma^{5}\left[\cos \left(k_{1}\right)+\cos \left(k_{2}\right)+\cos \left(k_{3}\right)+\cos \left(k_{4}\right)-3\right]
\end{aligned}
$$

in the momentum $\boldsymbol{k}$ space, where $\Gamma^{1}=\sigma^{1} \otimes \sigma^{3}, \Gamma^{2}=\sigma^{2} \otimes$ $\sigma^{3}, \Gamma^{3}=\sigma^{3} \otimes \sigma^{1}, \Gamma^{4}=\sigma^{0} \otimes \sigma^{2}$, and $\Gamma^{5}=\sigma^{0} \otimes \sigma^{3}$, which obey $\left\{\Gamma^{i}, \Gamma^{j}\right\}=2 \delta_{i j}$. If the $4 \mathrm{D}$ lattice is formed by two layers of 3D cubic lattices, the one-body Hamiltonian in the $\left(k_{1}, k_{2}, k_{3}\right)$ space is given by the following $8 \times 8$ matrix:

$$
\begin{aligned}
H_{3 \mathrm{D}}\left(k_{1}, k_{2}, k_{3}\right)= & \left(\begin{array}{ll}
M_{1} & M_{2} \\
M_{2}^{\dagger} & M_{1}
\end{array}\right), \quad \text { where } \\
M_{1}= & 2\left[\Gamma^{1} \sin \left(k_{1}\right)+\Gamma^{2} \sin \left(k_{2}\right)+\Gamma^{3} \sin \left(k_{3}\right)\right] \\
& +2 \Gamma^{5}\left[\cos \left(k_{1}\right)+\cos \left(k_{2}\right)+\cos \left(k_{3}\right)-3\right], \\
M_{2}= & -\mathrm{i} \Gamma^{4}+\Gamma^{5} .
\end{aligned}
$$

One can directly check that the above 3D fermion hopping model gives rise to a two-component massless complex Weyl fermion on each of the two 3D surfaces of the 4D lattice. The Weyl fermion on one boundary is a left-hand Weyl fermion and on the other boundary is a right-hand Weyl fermion. We have a similar result when the 4D lattice is formed by many layers of 3D cubic lattices.

The 16 copies of the local lattice model (7) give rise to the $3+1 \mathrm{D}$ Weyl fermions in the 16-dimensional spinor representation of the Spin(10) on the lattice boundary's lowenergy spectrum. The ground state of the $4+1 \mathrm{D}$ hopping model is

(a) a "lattice invertible topological state (invertible topological order whose low energy is an iTQFT) with a $\operatorname{Spin}(10) \supset$ $\mathbb{Z}_{2}^{f}$ symmetry," since it has no nontrivial topological excitations;

(b) a lattice nontrivial $4+1 \mathrm{D} \operatorname{Spin}(10)$ noninteracting free fermionic SPT state [56-58], which belongs to the 16-th class (or the $16 n$-th) in the $\mathbb{Z}$ classification.

But such a state may correspond to

(c) a trivial state for Spin(10) SPT state in the lattice interacting fermionic SPT systems [49,52,59-61] and to a trivial cobordism class (in 1, 2, and 3) [29,30], which belongs to the zeroth class in the classification.

If so, the $4+1 \mathrm{D}$ hopping model can have a symmetric gapped boundary, and the $3+1 \mathrm{D}$ Spin(10) chiral Weyl fermions (5) for the mirror sector can be gapped by interactions without breaking the symmetry by Propositions 1 and 2 .

To show that the $4+1 \mathrm{D}$ hopping model gives rise to a trivial Spin(10) SPT state in interacting fermion systems, we use a recent conjectured complete classification of interacting fermionic invertible topological orders [29,30,49,50,61-63] with on-site symmetry, via a twisted version of the spin cobordism theory of Freed-Hopkin [29]. This classification includes all known interacting fermionic SPT states and all known interacting fermionic invertible topological orders on a lattice $[52,59,60,64]$.

We first note that for fermions with the full symmetry $G_{f} \supset \mathbb{Z}_{2}^{f}$ in the $D$ spacetime dimensions, they transform as $G=\frac{\operatorname{Spin}(D) \times G_{f}}{\mathbb{Z}_{2}^{f}}$ under the combined spacetime symmetry $G_{\text {spacetime }}=\operatorname{Spin}(D)$ rotation and the internal $G_{f}$ transformation, where a double-counted fermion parity symmetry $\mathbb{Z}_{2}^{f}$ is mod out. This shared normal subgroup $\mathbb{Z}_{2}^{f}$ is due to the fact that rotating a fermion by $2 \pi$ in the spacetime (namely, the spin statistics) gives rise to the same fermion parity minus sign for the fermion operator $\psi \rightarrow-\psi$.

To classify the iTQFT whose boundary can have a $3+1 \mathrm{D}$ Spin(10) chiral fermion theory, we focus on the following cobordism group:

$\Omega_{\frac{[\operatorname{spin}(D=5) \times \operatorname{Spin}(10)]}{\mathbb{Z}_{2}^{f}}}^{D=\operatorname{TP}_{D=5}}\left\{\frac{[\operatorname{Spin}(D=5) \times \operatorname{Spin}(10)]}{\mathbb{Z}_{2}^{f}}\right\}$.

More generally, we find that $4+1 \mathrm{D}$ fermionic invertible topological orders with $G_{f}=\operatorname{Spin}(N) \supset \mathbb{Z}_{2}^{f}$ on-site global symmetry for $N \geqslant 7$ are classified by the fifth cobordism group [30]:

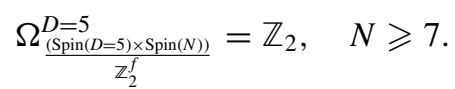

Note that we define the cobordism group, classifying symmetric fermionic invertible topological orders, as

$$
\begin{aligned}
\Omega_{G}^{D} & \left.\equiv \Omega_{\left({ }_{\left(G_{\text {spacetime }} \times G_{f}\right.}\right.}^{N_{\text {shared }}}\right) \\
& \equiv \operatorname{TP}_{D}(G) \equiv\left[M T(G), \Sigma^{D+1} I \mathbb{Z}\right],
\end{aligned}
$$

which stands for the homotopy classes of maps from the Thom-Madsen-Tillmann spectrum [43,44] $M T(G)$ to the $D+1$-th suspension of the Anderson dual spectrum $\Sigma^{D+1} I \mathbb{Z}$. Our notations follow Refs. [29,30,50] and [65]: TP abbreviates "topological phases" classifying the symmetric invertible topological orders (or invertible topological quantum field theories), and $N_{\text {shared }}$ is the shared normal subgroup of $G_{\text {spacetime }}$ and $G_{f}$.

The cobordism group of topological phases (TP) defined in [29] as $\operatorname{TP}_{D}(G)$ classifies the deformation classes of reflection positive invertible $d$-dimensional extended topological field theories with symmetry group $G_{D}$. The cobordism group $\mathrm{TP}_{D}(G) \equiv \Omega_{G}^{D}$ and the bordism group $\Omega_{D}^{G}$ are related by a 
short exact sequence,

$$
\begin{aligned}
0 & \rightarrow \operatorname{Ext}^{1}\left(\Omega_{D}^{G}, \mathbb{Z}\right) \rightarrow \mathrm{TP}_{D}(G) \\
& \equiv \Omega_{G}^{D} \rightarrow \operatorname{Hom}\left(\Omega_{D+1}^{G}, \mathbb{Z}\right) \rightarrow 0,
\end{aligned}
$$

with Ext denotes the extension functor; see Appendix E.

In contrast, we do not define the cobordism group as the usual definition of Pontryagin dual of the torsion subgroup ( $\equiv$ tors) of the bordism group $\Omega_{D}^{G}$ as the homomorphism (Hom) map to $\mathrm{U}(1)$ :

$$
\operatorname{Hom}\left[\Omega_{D}^{G \text {,tors }}, \mathrm{U}(1)\right] \text {, }
$$

although the torsion (i.e., finite group) sectors of (11) and (13) are equivalent. Mathematical details for the above result are presented in Refs. [30,50,55]. ${ }^{12}$ We classify the deformation classes of invertible topological quantum field theories (more precisely, the reflection positive invertible extended topological field theories) via $\Omega_{G}^{D}$, by classifying the cobordant differentiable and triangulable manifolds with a stable $G$ structure, via associating them to the homotopy groups of the Thom-Madsen-Tillmann spectra $[43,44]$, thanks to a theorem in Ref. [29].

To be precise, here the spin cobordism theory is believed to completely classify all the fermionic iTQFTs. By applying this spin cobordism theory, we classify $4+1 \mathrm{D} \operatorname{Spin}(N)$ SPT states and $4+1 \mathrm{D} \operatorname{Spin}(N)$ symmetric invertible fermionic topological orders. In fact, in this context, the $3+1 \mathrm{D} \operatorname{Spin}(N)$ fermion theories already include all possible $3+1 \mathrm{D} \operatorname{Spin}(N)$ chiral fermion theories that we need. To this end, we will especially focus on the $3+1 \mathrm{D} \operatorname{Spin}(10)$ chiral fermion theories with Weyl fermions in a 16-dimensional spinor representation.

The above $\mathbb{Z}_{2}$ classification in (10) implies that there is only one nontrivial $4+1 \mathrm{D}$ invertible fermionic topological order with a $\operatorname{Spin}(N)$ on-site symmetry. We find that such a topological phase is characterized by a five-dimensional topological invariant [30] written in terms of a bulk partition function on a 5-manifold $M^{5}$,

$$
\mathbf{Z}=e^{i \pi \int_{M^{5}} \mathrm{w}_{2}(T M) \cup \mathrm{w}_{3}(T M)},
$$

where $\mathrm{w}_{n}(T M)$ is the $n$ th-Stiefel-Whitney class for the tangent bundle of $4+1 \mathrm{D}$ spacetime manifold $M^{5}$, and the $U$ is the cup product (which we may omit writing $\cup$ ) [68].

We note that on $M^{5}$, we have a $\frac{\operatorname{Spin}(D=5) \times \operatorname{Spin}(N)}{\mathbb{Z}_{2}^{f}}$ connection - a mixed gravitational and gauge connection, rather than a pure gravitational $\operatorname{Spin}(D=5)$ connection, such that $\mathrm{w}_{2}(T M)=\mathrm{w}_{2}\left(V_{\mathrm{SO}(N)}\right)$ and $\mathrm{w}_{3}(T M)=\mathrm{w}_{3}\left(V_{\mathrm{SO}(N)}\right)$, where $\mathrm{W}_{n}\left(V_{\mathrm{SO}(N)}\right)$ is the $n$ th-Stiefel-Whitney class for an $\mathrm{SO}(N)$ gauge bundle. ${ }^{13}$ Namely, we find that there is only

\footnotetext{
${ }^{12}$ In contrast, Ref. [66] computes a different bordism group $\Omega_{D=5}^{[\mathrm{Spin}(D=5) \times \operatorname{Spin}(10)]}=0$. Instead, we study the bordism group $\Omega_{D=5}^{[\operatorname{Spin}(D=5) \times \operatorname{Spin}(10)] / \mathbb{Z}_{2}^{f}}=\mathbb{Z}_{2}$, whose manifold generator can detect the new $\mathrm{SU}(2)$ anomaly [67].

${ }^{13}$ In the context of anomalies (see Appendix A for details), the boundary of this $4+1 \mathrm{D} \operatorname{Spin}(N)$-SPT state may have a mixed anomaly of $\mathrm{SO}(N)$-gauge bundle and spacetime geometry/gravity, and we can use the this $3+1 \mathrm{D}$ anomaly on the boundary to detect the bulk invertible topological order.
}

one possible candidate of the $3+1 \mathrm{D}$ anomaly for interacting fermion systems with a $\operatorname{Spin}(N)$ symmetry $(N \geqslant 7)$, which is a nonperturbative global mixed gauge-gravity (i.e., gaugediffeomorphism) anomaly characterized by (14). Thus, $M^{5}$ may not be a spin manifold [note that a spin manifold requires $\mathrm{w}_{2}(T M)=0$ ], generally called a nonspin manifold.

We can detect the $4+1 \mathrm{D}$ cobordism invariant $e^{i \pi \int_{M^{5}} \mathrm{w}_{2}(T M) \mathrm{w}_{3}(T M)}$ for the $4+1 \mathrm{D}$ invertible fermionic topological order by study its boundary state. In particular, if the $4+1 \mathrm{D}$ state has a boundary described by $3+1 \mathrm{D}$ $\operatorname{Spin}(N)$ chiral Weyl fermion theory, then we can detect the $4+1 \mathrm{D}$ cobordism invariant via the $\operatorname{Spin}(N)$ representation of the chiral Weyl fermions on the boundary. Here we use a fact that the $4+1 \mathrm{D}$ cobordism invariant can be detected by restricting to a $\operatorname{SU}(2)=\operatorname{Spin}(3)$ subgroup of $\operatorname{Spin}(N)$ [67]: Let $n_{j}$ be the number of isospin- $j$ representations of $\mathrm{SU}(2)=\operatorname{Spin}(3) \subseteq \operatorname{Spin}(N)$ for $3+1 \mathrm{D}$ boundary chiral Weyl fermions; then the $4+1 \mathrm{D}$ cobordism invariant $e^{i \pi \int_{M^{5}} \mathrm{w}_{2}(T M) \mathrm{w}_{3}(T M)}$ is absent if

$$
\sum_{r=0}^{\infty} n_{2 r+\frac{1}{2}} \in \mathbb{Z}_{\text {even }}, \quad \sum_{r=0}^{\infty} n_{4 r+\frac{3}{2}} \in \mathbb{Z}_{\text {even }} .
$$

To see how the representation of $\operatorname{Spin}(N)$ reduces to the representations of $\mathrm{SU}(2)=\mathrm{Spin}(3)$, let us describe the representation of $\operatorname{Spin}(N)$ [the spinor representation of $\operatorname{Spin}(N)$ ], assuming $N=$ even. We first introduce $\gamma$-matrices $\gamma_{a}, a=$ $1, \ldots, N$ :

$$
\begin{aligned}
\gamma_{2 k-1} & =\underbrace{\sigma^{0} \otimes \cdots \otimes \sigma^{0}}_{\frac{N}{2}-k \sigma^{0} \mathrm{~s}} \otimes \sigma^{1} \otimes \underbrace{\sigma^{3} \otimes \cdots \otimes \sigma^{3}}_{k-1 \sigma^{3} \mathrm{~s}}, \\
\gamma_{2 k} & =\underbrace{\sigma^{0} \otimes \cdots \otimes \sigma^{0}}_{\frac{N}{2}-k \sigma^{0} \mathrm{~s}} \otimes \sigma^{2} \otimes \underbrace{\sigma^{3} \otimes \cdots \otimes \sigma^{3}}_{k-1 \sigma^{3} \mathrm{~s}},
\end{aligned}
$$

$k=1, \ldots, \frac{N}{2}$, which satisfy $\left\{\gamma_{a}, \gamma_{b}\right\}=2 \delta_{a b}$ and $\gamma_{a}^{\dagger}=\gamma_{a}$. Here, $\sigma^{0}$ is the $2 \times 2$ identity matrix and $\sigma^{l}$ with $l=1,2,3$ are the Pauli matrices. The $\frac{N(N-1)}{2}$ Hermitian matrices $\gamma_{a b}=$ $\frac{i}{2}\left[\gamma_{a}, \gamma_{b}\right]=i \gamma_{a} \gamma_{b}, a<b$, generate a $2^{N / 2}$-dimensional representation of $\operatorname{Spin}(N)$. The above $2^{N / 2}$-dimensional representation is reducible. To obtain an irreducible representation, we introduce

$$
\gamma_{\mathrm{FIVE}}=(-i)^{N / 2} \gamma_{1} \cdots \gamma_{N}=\underbrace{\sigma^{3} \otimes \cdots \otimes \sigma^{3}}_{\frac{N}{2} \sigma^{3}, \mathrm{~s}} .
$$

We have $\left(\gamma_{\text {FIVE }}\right)^{2}=1$, its trace $\operatorname{Tr}\left(\gamma_{\text {FIVE }}\right)=0$, and $\left\{\gamma_{\mathrm{FIVE}}, \gamma_{a}\right\}=\left[\gamma_{\mathrm{FIVE}}, \gamma_{a b}\right]=0$. This allows us to obtain two $2^{N / 2-1}$-dimensional irreducible representations: one for $\gamma_{\mathrm{FIVE}}=1$ and the other for $\gamma_{\mathrm{FIVE}}=-1$.

Now, let us consider an $\mathrm{SU}(2)=\operatorname{Spin}(3)$ subgroup of $\operatorname{Spin}(N)$, generated by $\gamma_{12}=I \otimes \sigma^{0} \otimes \sigma^{3}, \quad \gamma_{23}=I \otimes$ $\sigma^{1} \otimes \sigma^{1}$, and $\gamma_{31}=I \otimes \sigma^{1} \otimes \sigma^{2}$. We see that the $2^{N / 2-1}$. dimensional representation of $\operatorname{Spin}(N)$ becomes $2^{N / 2-2}$ isospin-1/2 representations of $\mathrm{SU}(2)$.

Summarizing the above results, we see that the $2^{N / 2-1}$ copies of $4+1 \mathrm{D}$ hopping model (7) formed by many (but finite) layers of $3 \mathrm{D}$ cubic lattices has a $3+1 \mathrm{D}$ boundary chiral Weyl fermion in the $2^{N / 2-1}$-dimensional representation of $\operatorname{Spin}(N)$ on one boundary (the normal sector), and a conjugate $3+1 \mathrm{D}$ chiral Weyl fermion on the other boundary (the 
mirror sector). For an even $N \geqslant 8$, the $3+1 \mathrm{D}$ boundary chiral Weyl fermions only reduce to an even number of isospin-1/2 representations, and, according to (15), the $4+1 \mathrm{D}$ cobordism invariant $e^{i \pi \int_{M^{5}} \mathrm{w}_{2}(T M) \mathrm{w}_{3}(T M)}$ is absent. Thus the corresponding $4+1 \mathrm{D}$ bulk state is a trivial fermionic iTQFT (i.e., the identity element as the trivial cobordism class in Propositions 1 and 2) with a $\operatorname{Spin}(N)$ symmetry.

In this case, the mirror sector can be chosen as a $\operatorname{Spin}(N)$ symmetric gapped boundary (Proposition 1), or the mirror sector can be gapped out without breaking the $\operatorname{Spin}(N)$ symmetry by introducing a proper symmetric fermion interaction on the boundary (Proposition 2). Since the $4+1 \mathrm{D}$ hopping model has many layers and the $3+1 \mathrm{D}$ boundary massless chiral Weyl fermion has no symmetric relevant deformation operators [in the renormalization group sense, e.g., there is no $\operatorname{Spin}(N)$ symmetric mass term], the symmetric interaction in the mirror sector on one boundary will not affect the lowenergy dynamics of the $3+1 \mathrm{D}$ massless chiral Weyl fermion in the normal sector on the other boundary. ${ }^{14}$

Now we apply a well-known lattice method. Finitewidth/layer lattice dimensional reduction:

An $n+1 \mathrm{D}$ lattice model with finite layers along one extra direction (a finite width $w$ ) can be dimensionally reduced to an $n \mathrm{D}$ lattice model via absorbing the degrees of freedom along $\mathrm{w}$ to the orbital in $n \mathrm{D}$.

Thus, by (18), the $4+1 \mathrm{D}$ hopping model with finite layers can be viewed as a $3+1 \mathrm{D}$ lattice model with finite orbitals per site, and the $3+1 \mathrm{D} \operatorname{Spin}(N)$ chiral Weyl fermion theory in the $2^{N / 2-1}$-dimensional representation can be regularized by a lattice model in the same dimension without breaking the $\operatorname{Spin}(N)$ symmetry for even $N \geqslant 8$.

In particular, for $N=10$, the $\operatorname{Spin}(10)$ chiral fermion theory with Weyl fermions in a 16-dimensional spinor representation [similarly, for Spin(18), chiral fermion theory in a 256-dimensional spinor representation] can be regularized by a local lattice fermion model in the same dimension. After regularizing the Spin(10) chiral fermion theory as a lattice fermion model in the same dimension $(3+1 \mathrm{D})$ with an onsite Spin(10) symmetry, we can gauge the on-site Spin(10) symmetry to obtain a gauged $\operatorname{Spin}(10)$ chiral fermion theory, ${ }^{15}$ again regularized by a lattice model in the same dimension.

\footnotetext{
${ }^{14}$ In the previous paragraph, we had determined that the $4+1 \mathrm{D}$ hopping model (7) without higher-order interactions, whose boundary hosts a $3+1 \mathrm{D}$ chiral Weyl fermion in the $2^{N / 2-1}$-dimensional representation of $\operatorname{Spin}(N)$, has a trivial cobordism class in the bulk. Readers may wonder whether the bulk's cobordism class would change under the interactions that we required. The answer is no. To recall, our setup follows: (i) Bulk does not include nonperturbative interactions. Bulk only allows small perturbative interactions if any. So the bulk gap does not close, and can never be closed. (ii) Only on the boundary, we can add "arbitrary $\operatorname{Spin}(N)$ preserving interactions" (both small perturbative or large nonperturbative interactions). In summary, since the bulk gap does not close, the bulk phase remains the same trivial cobordism class, which stays valid before and after adding boundary interactions.

${ }^{15}$ To gauge the on-site symmetry, one way is by inserting gauge variables on the one-dimensional links between local sites. This is
}

We remark that, in fact, for $N=3$, the $4+1 \mathrm{D}$ fermionic invertible topological orders with a $\operatorname{Spin}(3)=\mathrm{SU}(2)$ internal global symmetry are classified by the cobordism group of $[\operatorname{Spin}(D=5) \times \operatorname{Spin}(N=3)] / \mathbb{Z}_{2}^{f}[30,67]$ :

$$
\Omega_{\frac{[\operatorname{Sppin}(D=5) \times \operatorname{Spin}(3)]}{D=5}}^{\mathbb{Z}_{2}^{f}}=\Omega_{\frac{[\operatorname{lSpin}(D=5) \times \operatorname{SU}(2)]}{D=5}}^{\mathbb{Z}_{2}^{f}}=\left(\mathbb{Z}_{2}\right)^{2} .
$$

The corresponding cobordism invariant is given by

$$
\mathbf{Z}=e^{i \alpha \pi \int_{M^{5}} \operatorname{Arf} \widetilde{\mathrm{w}}_{3}(T M)} e^{i \beta \pi \int_{M^{5}} \mathrm{w}_{2}(T M) \mathrm{w}_{3}(T M)} .
$$

Here, Arf is the Arf invariant [69], which characterizes the $1+1 \mathrm{D}$ fermionic chain whose open ends host Majorana zero modes [70]. This $1+1 \mathrm{D}$ fermionic chain is also known as the Kitaev chain [70], whose low-energy physics is governed by a $1+1 \mathrm{D}$ invertible fermionic topological order. The $\widetilde{\mathrm{w}}_{3}(T M)$ is a twisted version of the third-Stiefel-Whitney class $\mathrm{w}_{3}(T M)$. The above cobordism invariant can be detected by the SU(2) representations of $3+1 \mathrm{D}$ boundary chiral Weyl fermions, and $\alpha, \beta$ in (20) are given by [67]

$$
\alpha=\sum_{r=0}^{\infty} n_{2 r+\frac{1}{2}} \bmod 2, \quad \beta=\sum_{r=0}^{\infty} n_{4 r+\frac{3}{2}} \bmod 2 .
$$

In this work, we only suggest that there exists a symmetric short-range nonperturbative interaction that can fully gap out the mirror sector without breaking the Spin(10) symmetry. ${ }^{16}$ Our approach only proves the symmetric gapped boundary exists (via Propositions 1 and 2), but does not provide a prescription to design such an interaction. The approach in Refs. [23,71] proposes a design: The interaction in the mirror sector is given by the smooth orientation fluctuations of Higgs field (thus beyond the Higgs mechanism [18,72]), where a constant orientation will gap out all the mirror fermions. But the validity of the design requires confirmation by numerical

known as the hard gauge, such that the outcome gauge theory does not have a tensor product Hilbert space (Definition 1), and thus it is not a local lattice model that we aim for. However, we can further maintain a tensor product Hilbert space (Definition 1) by designing the soft gauge. We relegate the details of the soft gauge via a local lattice model in Appendix B. See discussions on hard gauge and soft gauge in Ref. [18].

${ }^{16}$ There exists such a symmetric gapping interaction preserving Spin(10). Moreover, for 16 chiral Weyl fermions at IR, there can be a U(16) global symmetry, with $U(16) \supset S$ pin(10). It is possible that additional constraints happen on what interactions we can engineer in the quotient space $\frac{\mathrm{U}(16)}{\operatorname{Spin}(10)}$ (also a homogeneous space) without breaking Spin(10). We provide some further guidelines for designing interactions: (a) To gap the mirror sector by adding nonperturbative lattice-scale interactions on the boundary, we may need to look for a larger symmetry $G_{+}$than $\operatorname{Spin}(10)$, such that

$$
\operatorname{Spin}(10) \subseteq G_{+} \subseteq \mathrm{U}(16) .
$$

This $G_{+}$can be useful for constructing exactly solvable and integrable models. (b) However, we can further weakly break $G_{+}$down to Spin(10), by small perturbative interactions that are not merely irrelevant perturbations in the renormalization group (RG) and field theory sense, but also with small lattice-scale couplings. Thus we can break the redundant symmetry outside Spin(10) without changing the quantum dynamics. 
simulations. A first step is taken in Ref. [71] for a 1 + 1D system. In such a design, crucially, the mass of the mirror fermions induced by the Higgs field must be comparable with the fermion bandwidth. Some other gapping-mirror-fermion approaches have also been proposed recently [17,72-77]. Many previous calculations [26,27] checking the mirror fermion approach choose an induced energy gap (i.e., an effective mass) to be much bigger than the bandwidth (i.e., at the infinite coupling limit). The infinite coupling limit in the mirror sector generates a dead layer; a neighbor layer next to the mirror sector would become the new mirror sector with fermion doublings [8], which would fail to produce a chiral fermion/gauge theory at low energies.

\section{SU(5) CHIRAL FERMION THEORY AND SU(5) GRAND UNIFICATION}

Above we have discussed the lattice regularization of a Spin(10) gauged chiral fermion theory. To consider a lattice regularization of a $\mathrm{SU}(5)$ gauged chiral fermion theory [with $G_{f}=\mathbb{Z}_{2}^{f} \times \mathrm{SU}(5)$, but only SU(5) will be gauged], we classify the $4+1 \mathrm{D}$ invertible fermionic topological order with $G_{f}=\mathbb{Z}_{2}^{f} \times \mathrm{SU}(5)$ symmetry by a cobordism group defined in Eq. (11) [note that $\left.\operatorname{Spin}(D=5) \supset \mathbb{Z}_{2}^{f}\right][30,55]$ :

$$
\Omega_{\mathrm{Spin}(D=5) \times \mathrm{SU}(5)}^{D=5} \equiv \mathrm{TP}_{D}[\operatorname{Spin}(D=5) \times \mathrm{SU}(5)]=\mathbb{Z},
$$

where the topological invariant is given by the SU(5) ChernSimons 5-form, associated with perturbative local anomalies captured by perturbative Feynman diagram calculations in $3+1 \mathrm{D}$.

Again, such a cobordism invariant and the associated invertible topological order can be detected by the boundary chiral fermions: if the $3+1 \mathrm{D}$ boundary SU(5) chiral fermion theory is free from any of the $\mathbb{Z}$ class of $S U(5)$ perturbative local anomaly, then the corresponding cobordism invariant and the $4+1 \mathrm{D}$ bulk invertible topological order are trivial. Thus, by Propositions 1 and 2, any SU(5) gauged chiral fermion theory that can be regularized at the boundary of a $4+1 \mathrm{D}$ gapped local lattice model can be regularized by a $3+$ 1D local lattice model via the method (18), provided that the $\mathrm{SU}(5)$ gauge theory is free of the $\mathrm{SU}(5)$ perturbative anomalies (see, also, Proposition 4 in Appendix A 1). In particular, the SU(5) grand unified theory [5] can be regularized by a lattice. This implies that its induced 15 -fermion standard model can be regularized by a lattice fermion model.

\section{IMPLICATIONS AND CONCLUSIONS}

In fact, an $n+1 \mathrm{D} G$-symmetric iTQFT given by a cobordism class in Propositions 1 and 2 corresponds to an $n \mathrm{D}$ 't Hooft anomaly of $G$-symmetry (see footnote 4 and the details of anomalies in Appendix A). So a trivial cobordism class in $n+1 \mathrm{D}$ for $G$-symmetry means all-'t Hooft-anomaly-free in $n \mathrm{D}$ for the full $G$-symmetry. Namely, by far we only show that anomaly-free gauged chiral fermion theories can be defined on a lattice with nondynamical background gauge fields (Models 1 and 2 in Appendix A 1), regularized with on-site symmetries in its own dimensions [via (18)]. However, we can obtain a dynamical chiral gauge theory (Model 3 in Appendix A 1) by dynamically gauging the on-site symmetry: introducing dynamical gauge link variables between local sites (e.g., dynamically sum over gauge inequivalent configurations in the partition function)- this is a hardgauge model but not a local lattice model; see footnote 15 . We can further apply the soft-gauge method [18] to obtain a local lattice model; see Appendix B. We emphasize if all gauge-invariant operators are bosonic, the above dynamical lattice gauge theory coupled to fermions is actually a local lattice bosonic model in disguise, as one can see from the slave-particle/parton approach [36,78-81].

We remark that the dynamical Spin(10) chiral gauge theory coupled to Weyl fermions in the 16-dimensional spinor representation is a local bosonic theory, since all gauge-invariant operators are bosonic. ${ }^{17}$ The lattice regularization that realizes the dynamical Spin(10) chiral gauge theory is also a local bosonic model (see Appendix B). In other words, the Spin(10) dynamical chiral gauge theory with Weyl fermions in a 16dimensional representation, and the induced 16-fermion standard model, can be regularized as the low-energy effective theory of a local lattice model of qubits (since any local bosonic lattice model can be viewed as a lattice model of qubits). Based on the stability of cobordism group of Eq. (10) for $N \geqslant 7$, our result directly applies to a $\operatorname{Spin}(N=18)$ chiral gauge theory $[72,82]$, which is also a local bosonic model. Thus our study implies that all elementary particles (except the graviton) can be viewed as originated from qubits [83-85]. It is a concrete realization of "it from qubit [86]," representing an ultra unification of all gauge interactions and matter fermions in term of quantum information (i.e., qubits).

The statement that all elementary particles arise from bosonic qubits has a falsifiable experimental prediction: all fermions and their fermionic bound states must carry nontrivial gauge charge [36,87]. As a result, the "standard model" from a lattice qubit model cannot just have a $\frac{\mathrm{U}(1) \times \mathrm{SU}(2) \times \mathrm{SU}(3)}{\mathbb{Z}_{q}}$ gauge group, since such a standard model indeed has fermionic bound states that carry no gauge charge. Thus, the "standard model" from a lattice qubit model must have a larger gauge group, e.g., adding a new $\left[\mathbb{Z}_{2}\right]$ gauge sector, ${ }^{18}$ where we gain a new cosmic string (whose spacetime

\footnotetext{
${ }^{17}$ In Appendix B, we provide the explicit slave-particle/parton construction for a $4+1 \mathrm{D}$ local bosonic lattice model, whose boundary can give rise to the dynamical Spin(10) chiral gauge theory coupled to Weyl fermions (Model 3) in the 16-dimensional representation.

${ }^{18}$ See Footnote 11; we can show that

$$
\begin{aligned}
\frac{\operatorname{Spin}(D) \times \operatorname{Spin}(10)}{\mathbb{Z}_{q^{\prime}}} \supset & \operatorname{Spin}(D) \times \operatorname{SU}(5) \supset \operatorname{Spin}(D) \\
& \times \frac{\mathrm{U}(1) \times \operatorname{SU}(2) \times \operatorname{SU}(3)}{\mathbb{Z}_{6}},
\end{aligned}
$$

where $q^{\prime}=1$ or 2 , while

$$
\mathrm{SO}(10) \supset \mathrm{SU}(5) \supset \frac{\mathrm{U}(1) \times \mathrm{SU}(2) \times \mathrm{SU}(3)}{\mathbb{Z}_{6}}
$$

and$$
\operatorname{Spin}(10) \supset \mathrm{SU}(5) \supset \frac{\mathrm{U}(1) \times \mathrm{SU}(2) \times \mathrm{SU}(3)}{\mathbb{Z}_{6}} .
$$

For $q^{\prime}=2$, when we gauge the [Spin(10)], we also require one to gauge the $\left[\mathbb{Z}_{2}^{f} \times \mathrm{SU}(5)\right]$ in the embedded smaller group $\operatorname{Spin}(D) \times$
} 
trajectory is a two-dimensional worldsheet) - the flux line of the new $\left[\mathbb{Z}_{2}\right]$ gauge field [88].

In contrast, the dynamical SU(5) chiral gauge theory coupled to Weyl fermions in the five- and 10-dimensional representations is a fermionic theory definable on spin manifolds, since some gauge-invariant operators are fermionic. The lattice regularization that realizes the dynamical SU(5) chiral gauge theory is also a local fermionic model (which is not a local lattice model of qubits). The standard model from local fermionic lattice models can have $\frac{\mathrm{U}(1) \times \mathrm{SU}(2) \times \mathrm{SU}(3)}{\mathbb{Z}_{q}}$ as its gauge group; see footnote 18. It does not require extra gauge groups.

In our work, we have shown that a Spin(10) [or SU(5)] chiral gauge theory with 16 (or 15) Weyl fermions can be regularized by a lattice, since the mirror sector can be fully gapped by Spin(10) [or SU(5)] symmetric interactions without spontaneously breaking the symmetry. However, it is possible that the mirror sector can be fully gapped by interactions with a larger symmetry $G_{+}$without spontaneously breaking the symmetry $G_{+}$. In this case, after gauging Spin(10) [or $\mathrm{SU}(5)]$, the chiral gauge theory may have an exact global symmetry $G_{Q}$ (on a lattice scale or a UV cutoff scale such as an effective Planck scale) sitting as a quotient group satisfying the short exact sequences:

$$
\begin{aligned}
1 \rightarrow \operatorname{Spin}(10) & \rightarrow G_{+} \rightarrow G_{Q} \rightarrow 1, \\
\text { or } \quad 1 & \rightarrow \mathrm{SU}(5) \rightarrow G_{+} \rightarrow G_{Q} \rightarrow 1 .
\end{aligned}
$$

The reason $G_{Q}$ is still a global symmetry after gauging $\operatorname{Spin}(10)$ [or SU(5)] is that because $G_{+}$can be chosen as an on-site symmetry on the UV cutoff scale and $G_{+}$is anomaly free on the $d+1 \mathrm{D}$. Once a normal subgroup is gauged, the $G_{Q}$ is still anomaly free and unbroken, and thus can still be made on-site in $d+1 \mathrm{D}$.

Lastly, we comment on the dynamics of these dynamical chiral gauge theories (Model 3, as highly long-range entangled states). At the low energy of these chiral gauge theories, there could be emergent symmetries (e.g., higherform symmetries [33] or higher symmetries in general [30]) having new 't Hooft anomalies. However, emergent new anomalies only mean the emergent symmetries cannot be strictly regularized locally on-site, on-link, on- $n$-simplex, etc., which, we emphasize, is a rather distinct issue deviated from regularizing chiral fermion theories which we solved earlier. After regularizing chiral fermion theories on a lattice and after dynamically gauging, the emergent new anomalies only constrain the dynamics of gauge theories (e.g., gapless near a quantum critical fixed point, or emergent symmetry spontaneously broken, etc). We aim to address the dynamics of gauge theories in future work.

\section{ACKNOWLEDGMENTS}

We thank E. Witten for many helpful discussions and comments, and for a collaboration on Ref. [67]. J.W. thanks Z. Wan for related collaborations. J.W. is supported by Corning Glass Works Foundation Fellowship and NSF Grant No.

$\operatorname{SU}(5) \subset \frac{\operatorname{Spin}(D) \times \operatorname{Spin}(10)}{\mathbb{Z}_{q^{\prime}=2}}$. The dynamically gauging $\left[\mathbb{Z}_{2}^{f}\right]$ symmetry produces the new $\left[\mathbb{Z}_{2}\right]$ gauge sector.
PHY-1606531. X.-G.W. is partially supported by NSF Grant No. DMS-1664412. This work is also supported by NSF Grants No. PHY-1306313, No. PHY-0937443, No. DMS1308244, No. DMS-0804454, and No. DMS-1159412, and Center for Mathematical Sciences and Applications at Harvard University.

\section{APPENDIX A: DEFINITIONS OF TERMINOLOGY AND DISCUSSIONS BASED ON ANOMALIES}

In the main text, we have described our results without directly mentioning the quantum anomaly (footnote 4). However, in the literature, many people discuss the gauge chiral fermion problem in terms of anomaly. In this section, we will discuss our approach using the concept of the anomaly. We will carefully define several different anomalies. We will also carefully define several concepts of chiral fermion field theory and the concepts of lattice theory as a well-defined quantum theory.

\section{Detailed definitions of some relevant concepts}

We should clarify several related concepts of Spin(10) chiral fermion field theories and models as follows:

Model 1. Without gauging or before gauging Spin(10) symmetry, the theory is a "Spin(10) chiral fermion theory" with the full internal global symmetry $G_{f}=\operatorname{Spin}(10) \supset \mathbb{Z}_{2}^{f}$. In this case, we call the anomaly associated with the global symmetry $G_{f}$ as the 't Hooft anomaly of $G_{f}$. We classify the 't Hooft anomaly [89] of $G_{f}$ in Eq. (10) and Eq. (11).

Model 2. We may twist the Spin(10) symmetry via a nondynamical background Spin(10) gauge field, known as the symmetry twist. We name such a theory as a "Spin(10) gauged chiral fermion theory." The anomaly of $G$ classified in (10) becomes the background gauge anomaly, which is the same as the 't Hooft anomaly in nature.

Model 3. After dynamical gauging Spin(10) symmetry via dynamical weakly fluctuating $\operatorname{Spin}(10)$ gauge field, the theory becomes a "Spin(10) chiral gauge theory." In this case, it is a standard terminology to call the anomaly, descending from Model 1's 't Hooft anomaly and after gauging Spin(10), as the dynamical gauge anomaly. We will thus also study the dynamical gauge anomaly of Spin(10), thanks to Eq. (10). If any model possesses any dynamical gauge anomaly, then this theory is inconsistent and thus ill defined.

Reference [23] adopted a different viewpoint (or a different definition) of anomalies proposed in Ref. [90] (see Definition 7), for interacting quantum theories, which in turn leads to a classification of anomalies. Before we proceed, we should clarify some conventions of terminology as the definitions:

Definition 1. Well-defined quantum theories are quantum theories defined with a finite-dimensional Hilbert space and a finite-dimensional Hamiltonian matrix for a finite-size system in the real space. In this work, we only focus on this class of quantum theories.

Local lattice models are interacting or noninteracting lattice models whose many-body Hilbert space $\mathcal{V}$ has the following tensor product decomposition:

$$
\mathcal{v}=\bigotimes_{i} \mathcal{v}_{i}
$$


where $\mathcal{V}_{i}$ is a finite-dimensional Hilbert space for each lattice site.

By interacting models, we mean the Hamiltonian contains certain higher-order terms beyond the quadratic terms of fundamental lattice operators (such as quartic fermionic or spin operator terms beyond the quadratic terms).

By noninteracting models (or the so-called free or quadratic models), we mean the Hamiltonian contains, at most, the quadratic terms (thus easily diagonalizable and solvable) of fundamental lattice operators.

Local interactions. By local interactions, we mean that all interaction terms in the Hamiltonian (or a Lagrangian in the path integral) must be bounded by a finite range of lattice spacings. We call these types as local, finite-range, or shortrange interactions. We do not allow infinite-range interactions or the interactions with strength that exponentially decays to zero only at infinite. For any interaction term of our lattice model, it must be bounded by a finite spatial range; say, if the operators act on any site $i$ to $j$, then the locality means that " $|i-j| \leqslant$ a finite distance."

We emphasize that conventional lattice gauge theories with dynamical gauge fields are usually not local lattice models: Since there is a nonlocal gauge constraint, the tensor product decomposition (A1) is violated. In this work, we do not use models of conventional lattice gange theories, but limit ourselves to only local lattice models.

Definition 2. The on-site symmetry, for such local lattice models, is defined as a global internal symmetry, whose symmetry transformation operator has the following tensor product decomposition:

$$
U=\bigotimes_{i} U_{i}
$$

where $U_{i}$ is a unitary operator acting on $\mathcal{V}_{i}$.

Definition 3. Well-defined quantum field theory (living on the boundary of lattice model). When we mention a "welldefined" quantum field theory (QFT), we always mean a limited class of QFTs which can be regularized (i.e., regularized) as the low-energy effective boundary theory of a gapped local lattice model (see Definition 1) in one higher dimension (the so-called bulk). The global symmetry, if any, is regularized as an on-site symmetry (see Definition 2) for the full bulkboundary system. Such a QFT has, at most, the b-anomaly to be defined later in Definition 7. A "well-defined" QFT cannot have the r-anomaly that is defined later in Definition 8.

Definition 4. All lattice obstruction free (required to be regularizable in the same dimension). The above-defined QFTs include $d+1 \mathrm{D}$ QFTs that can be regularized by a lattice model in the same dimension $d+1 \mathrm{D}$ (with the symmetry, if any, regularized as an on-site symmetry or a local on- $n$ simplex symmetry ${ }^{19}$ ) because the gapped bulk in one higher dimension (of Definition 3) can be a decoupled gapped tensor

\footnotetext{
${ }^{19}$ Here we only focus on the well-defined $G$-symmetric QFTs with ordinary $G$-global symmetries (the 0 -form symmetry in the sense of generalized global symmetries [33]). If there is a generalized higher global symmetry [33], then we need to modify the "lattice on-site symmetry realization" to the "lattice local on- $n$-simplex symmetry realization."
}

product state. This leads to a concept of all lattice obstruction free: By saying a $d+1 \mathrm{D}$ QFT is all lattice obstruction free, we always mean a $d+1 \mathrm{D}$ well-defined quantum field theory in Definition 3, which can be regularized as the lowenergy effective boundary theory of a $d+2 \mathrm{D}$ gapped tensor product state (i.e., a gapped trivial vacuum) on a one-higherdimensional lattice. Note that a tensor product state (i.e., a trivial state, with neither short-range nor long-range entanglement of Definition 5) in a local lattice model is defined as

$$
|\Psi\rangle=\bigotimes_{i}\left|\psi_{i}\right\rangle, \quad\left|\psi_{i}\right\rangle \in \mathcal{V}_{i},
$$

which can be gapped and decoupled from its boundary theory. In contrast, the generic state is more general and is not necessarily a tensor product, such as

$$
|\Psi\rangle=\sum_{\left\{\mathrm{c}_{i \mathrm{i}\}}\right\}} \mathrm{c}_{\{\mathrm{i}\}}\left(\bigotimes_{\mathrm{i}}\left|\psi_{\mathrm{i}}\right\rangle\right), \quad\left|\psi_{\mathrm{i}}\right\rangle \in \mathcal{V}_{\mathrm{i}},
$$

with generic complex normalizable coefficients $\mathrm{c}_{\{\mathrm{i}\}}$.

Definition 5. Gapped system and entanglement. By a gapped tensor product state, we mean that the tensor product state is a unique ground state (with an energy $E_{0}$ ) of some lattice bulk Hamiltonian system whose energy spectrum has a finite energy gap $\Delta_{E}=E_{\text {excited }}-E_{0}>0$ separated from all excited states $E_{\text {excited }}$. Below the energy gap $\Delta_{E}$, the system behaves as a gapped trivial vacuum (or a gapped trivial insulator in condensed matter) with no entanglement.

On the other hand, general gapped systems $\left(\Delta_{E}=\right.$ $E_{\text {excited }}-E_{0}>0$ ) can generically possess short-range or longrange entanglements.

Short-range entangle states, short-range entanglements (SREs), and SPT states are defined as those gapped quantum ground states which can be deformed via local unitary transformations (LUTs) to a trivial tensor product state once we remove part of or all of the internal global symmetries [16]. Namely, along the deformations to a trivial tensor product state, the LUTs may break some internal global symmetry of the state. Gapped SRE states are also named SPT states.

Long-range entangle states, long-range entanglements (LREs), and topological orders are defined as those gapped quantum ground states which cannot be deformed via local unitary transformations (LUT) to a trivial tensor product state, even if we remove all internal global symmetries. Gapped LRE states are also named topological orders.

By this Definition 5, we can also rephrase Definition 4 as a well-defined quantum field theory (in Definition 3) is all lattice obstruction-free (Definition 4) if it can be regularized as the low-energy effective boundary theory of a gapped bulk lattice system whose bulk has no LRE (i.e., no topological order) and no SRE (i.e., no SPT state), and thus the bulk has no entanglement structure at all as a gapped trivial tensor product state. Readers should be cautious that although this gapped bulk alone has no entanglement, the boundary theory (such as an all-anomaly-free QFT) can be highly entangled and can have gapless states.

Definition 6. All anomaly free (i.e., here, free of all invertible bosonic and fermionic b-anomalies). The recent development suggests that all anomaly-free conditions of $d+1 \mathrm{D}$ $G$-symmetric QFT can be understood as the QFT can live 
on the boundary of a trivial cobordism class of a trivial invertible topological quantum field theories (iTQFT) from a corresponding cobordism group [29] or its higher-symmetry and higher-classifying space generalization [30-32]:

$$
\Omega_{G}^{d+2} \text {. }
$$

Namely, the trivial iTQFT is the trivial element 0 in the $\Omega_{G}^{d+2}$. Let us explain this development below.

The 't Hooft anomaly is a property in which the global symmetry of the theory cannot be made on-site on a lattice, and thus there is an obstruction to gauge the non-on-site symmetry, which is called the anomalous symmetry (Model 1) $[18,90]$. Dynamical gauge anomaly is a property in which its theory is ill defined (discussed in Model 3). How do we classify the property of non-on-site global symmetries or seemly ill-defined theories?

The previous anomaly inflow picture relates the anomalous noninteracting field theories or noninteracting lattice models (Definition 1) to the boundary of one-higher-dimensional bulk [91,92]. Reference [93] systematically described anomalies in field theories in terms of topological invariants in one higher dimension (such as the index of a Dirac operator), which turn out to be cobordism invariants [94]. However, to construct an interacting lattice regularization of a field theory, we need to classify anomalies in interacting field theories and interacting lattice models. Reference [90] attempts to classify anomalies in interacting lattice models, via topological orders and symmetry-protected topological states (SPTs) of interacting lattice models in one higher dimension.

Let us introduce a few different concepts of anomalies as the definitions of the terminology:

Definition 7. b-anomaly (三 boundary defined anomaly). There are anomalous theories that can be regularized as the low-energy effective boundary theory of a gapped local interacting lattice model in one higher dimension, where the global symmetry, if any, is regularized as an on-site symmetry for the whole bulk-boundary coupled system. However, the effective symmetry, if any, on the effective boundary theory alone is non-on-site. There is an obstruction to gauge the non-on-site symmetry $[18,90]$ because the standard gauging only works for an on-site symmetry: there is no canonical way to input the gauge variables on the links between "nonlocal sites" where the non-on-site symmetry acts. The obstruction of gauging is the same phenomenon that happened in 't Hooft anomalies. We will call this kind of anomalies the $b$-anomalies, which include the 't Hooft anomalies (associated with some internal global symmetry), gravitational anomalies (associated with no internal global symmetry), and their mixed anomalies.

Definition 8. r-anomalies (三 radical anomaly). There are also anomalous theories that cannot be regularized as the low-energy effective boundary theory of any gapped local lattice model in one higher dimension. We will call this kind of anomalies the r-anomalies, which include the dynamical gauge anomalies. A theory with an r-anomaly is simply an ill-defined quantum theory.

However, for a dynamical gauge theory with an r-anomaly, very often, we ungauge the theory to turn the dynamical gauge field (on the link or on $n$-simplex) into a global symmetry transformation [on-site or on $(n-1)$-simplex]. The resulting ungauged quantum theory may have a b-anomaly ('t Hooft anomaly) instead of an r-anomaly. (See examples below).

Definition 9. Invertible vs noninvertible anomalies. There are invertible anomalies that can be canceled by other anomalies. (The anomalies discussed in the field theory literature are mostly invertible anomalies). Invertible anomalies form an Abelian group, such as an infinite integer group $\mathbb{Z}$ (i.e., a perturbative local anomaly, captured by a Feynman diagram loop calculation) or a finite group $\mathbb{Z}_{n}$ of some positive integer $n$ (i.e., a nonperturbative global anomaly), or the product groups of $\mathbb{Z}$ and $\mathbb{Z}_{n}$. The invertible anomaly labeled by an Abelian group element $g$ can be canceled by an inverted anomaly labeled by an inverted Abelian group element $g^{-1}$. There are also noninvertible anomalies [41,90,95-98] that cannot be canceled by any other anomalies.

Definition 10. Bosonic vs fermionic anomalies. There are bosonic anomalies where the local operators in the corresponding anomalous theories are all bosonic [99,100]. There are fermionic anomalies where some local operators in the corresponding anomalous theories are fermionic. For example, a Spin(10) chiral Weyl fermion theory has an internal symmetry Spin(10) $\supset \mathbb{Z}_{2}^{f}$ containing the fermion parity, and thus we will need to classify possible fermionic anomalies of the interacting fermionic theory [later in Eq. (10)] in order to classify all of its anomalies. ${ }^{20}$

For more examples,

(a) A $1+1 \mathrm{D}$ chiral complex Weyl fermion theory with a Hamiltonian, $H=\mathrm{i} \psi^{\dagger} \partial_{x} \psi$, and a one-component complex Weyl spinor $\psi$, has

a fermionic invertible b-anomaly.

It is invertible because the anomaly has a $\mathbb{Z}$ class as a group classification.

(b) A $3+1 \mathrm{D}$ Weyl fermion doublet coupled to a probed (thus nondynamical) SU(2) background gauge field has the Witten SU(2) anomaly [101] as a type of 't Hooft anomaly of the SU(2) global symmetry, which is

$$
\text { a fermionic invertible b-anomaly. }
$$

It is fermionic because the $S U(2) \supset \mathbb{Z}_{2}^{f}$ has the fermion parity at its $\mathbb{Z}_{2}$ center. It is invertible because the anomaly has a $\mathbb{Z}_{2}$ class as a group classification.

(c) A $3+1 \mathrm{D}$ Weyl fermion doublet coupled to a dynamical SU(2) gauge field has the Witten SU(2) anomaly [101], which is

a bosonic invertible r-anomaly.

It is bosonic since all the local operators are gauge invariant and bosonic. Namely, the $\operatorname{SU}(2)\left(\supset \mathbb{Z}_{2}^{f}\right)$ is dynamically gauged, and thus the fermion parity $\mathbb{Z}_{2}^{f}$ is also gauged and the full theory is bosonic. It is an r-anomaly since the Weyl fermion coupled to this SU(2) gauge theory cannot be regularized as a boundary of any gapped local bosonic lattice model

\footnotetext{
${ }^{20}$ However, once the $[\operatorname{Spin}(10)] \supset \mathbb{Z}_{2}^{f}$ is gauged and thus the fermion parity $\mathbb{Z}_{2}^{f}$ is gauged in the $\operatorname{Spin}(10)$ chiral Weyl fermion theory, it becomes a Spin(10) chiral gauge theory, where all local gauge-invariant operators are bosons.
} 
[67]. However, if we ungauge the SU(2) of this ill-defined gauge theory, then its bosonic invertible r-anomaly (Definition 8 ) becomes the previous fermionic invertible b-anomaly.

(d) $\mathrm{A} \mathbb{Z}_{2}$ gauge theory in $2+1 \mathrm{D}$ or above with only $\mathbb{Z}_{2}$ charge excitations has

\section{a bosonic noninvertible b-anomaly,}

regularized as a boundary theory of a one-higher-dimensional $\mathbb{Z}_{2}$ gauge theory, which is a topological quantum field theory (TQFT).

The classification in Ref. [90] is a classification of all b-anomalies in terms of the topological orders [102] or symmetry-protected topological (SPT) states [15,16,46] in local lattice models in one higher dimension. A b-anomaly is invertible if it is characterized by an SPT state or an invertible topological order $[41,42,103,104]$ in one higher dimension. In this work, we will only focus on the invertible b-anomalies and their classifications.

From now on, by anomalous field theory, we will specifically mean a well-defined quantum field theory (Definition 3) with, at most, some invertible b-anomalies (defined in Definition 7). In this work, we only study well-defined quantum field theories (Definition 3) as the effective low-energy theory of the boundary of local lattice models (Definition 1). So, we exclude theories with the r-anomaly (defined in Definition 8) since they are not well-defined quantum theories (by the norm of both Definitions 1 and 7, and the standard lore).

According to the above classification, an anomaly-free (Definition 6) well-defined quantum field theory (Definition $3)^{21}$ is simply a boundary theory of a gapped trivial state (a tensor product state) on a one-higher-dimensional lattice, which means all lattice obstruction free that can also be regularizable in the same dimension (Definition 4).

The generalization of the anomaly inflow to a lattice model with interactions is crucial to obtain this result since some of the key concepts, such as the tensor product state and the onsite symmetry, require a lattice (providing the locality of sites) to define.

With the above terminology definitions, we claim a proposition:

Proposition 4. For any well-defined quantum field theory (Definition 3), if it is

\section{all anomaly free (Definition 6)}

with a list of conditions in footnote 21 , then it is

$$
\text { all lattice obstruction free (Definition 4), }
$$

which is required to be regularizable in the same dimension. Namely, any well-defined QFT that is all anomaly free can be regularized by a local interacting lattice model in the same dimension, where the global symmetry is regularized

\footnotetext{
${ }^{21}$ Thus, here the all-anomaly-free condition for a well-defined quantum field theory (Definition 3) specifically satisfies the following: (i) free of b-anomalies in Definition 7, (ii) free of all invertible anomalies in Definition 9, (iii) free of bosonic and fermionic anomalies in Definition 10.
}

as an on-site symmetry (or generalized local on- $n$-simplex symmetries) [90]. ${ }^{22}$

This result can be used to solve the gauged chiral fermion problem via the mirror fermion approach [23]: Given a $d+$ $1 \mathrm{D}$ gauged chiral fermion theory with a gauge group $G_{f} \supset$ $\mathbb{Z}_{2}^{f}$, we first ungauge, and obtain a $d+1 \mathrm{D}$ chiral fermion theory with an internal global symmetry group $G_{f} \supset \mathbb{Z}_{2}^{f}$. Then, we find a gapped $d+2 \mathrm{D}$ lattice model with a symmetry $G_{f} \supset$ $\mathbb{Z}_{2}^{f}$ whose boundary regularizes the ungauged $d+1 \mathrm{D}$ chiral fermion theory (Model 1). The symmetry $G_{f}$ is regularized as an on-site symmetry of the $d+2 \mathrm{D}$ lattice model. Next, we determine whether or not the ground state of the bulk gapped $d+2 \mathrm{D}$ lattice model has a trivial topological order and a trivial SPT state. If the $d+2 \mathrm{D}$ ground state indeed has no topological order and no SPT state (which is a trivial tensor product state by Definition 4), then the $d+1 \mathrm{D}$ ungauged chiral fermion theory can be regularized as the low-energy effective theory of a $d+1 \mathrm{D}$ local lattice model. Also, the $d+1 \mathrm{D}$ gauged chiral fermion theory can be regularized as the low-energy effective theory of a $d+1 \mathrm{D}$ local lattice model after gauging the on-site symmetry $G_{f}$.

To show the above claim, we can choose the $d+1 \mathrm{D}$ lattice model to be a slab of the $d+2 \mathrm{D}$ lattice model with a finite number of layers in the extra dimension. In such a model, the normal sector (or the chiral fermion sector) lives on one surface of the slab and the mirror fermion sector lives on the other surface of the slab. If the normal sector is free of all anomalies, it implies that the $d+2 \mathrm{D}$ bulk is actually a trivial gapped phase. If so, the mirror sector can be chosen to be a symmetric gapped boundary and can be fully gapped out without breaking the on-site symmetry $[15,16,18]$. A detailed explanation is given in Appendix A 2. Since the $d+2 \mathrm{D}$ slab has only finite layers, the $d+2 \mathrm{D}$ slab is actually a $d+1 \mathrm{D}$ lattice model with finite orbitals per site. Lastly, we gauge the on-site symmetry to obtain a gauged chiral fermion theory.

Thus, the above understanding suggests the following:

\footnotetext{
${ }^{22}$ See footnote 19 for the comment on the local symmetry realizations on the lattice. Above we propose the following:

If "all anomaly free" $\rightarrow$ then "all lattice obstruction free."

However, some well-defined QFTs (Definition 3) can be regularized on the boundary of one-higher-dimensional lattice model, e.g., even if they have b-anomalies in Definition 7. Thus, there is a subtlety about the converse statement. Only when we restrict the "all lattice obstruction-free" requiring QFT to be regularizable in the same dimension and all symmetries regularized strictly locally (Definition $4)$, is the converse statement also true:
}

$$
\text { If "all lattice obstruction free" } \rightarrow \text { then "all anomaly free." }
$$

In this work, when we classify invertible 't Hooft anomalies of global symmetries $G$, we use the cobordism group $\Omega_{G}^{d+2}$ in (A5) whose category of manifolds are only smooth and differentiable manifolds. Therefore, we can apply a known mathematical fact that all those smooth and differentiable manifolds are triangulable manifolds, via the Morse theory. Thus the anomalies captured in $\Omega_{G}^{d+2}$ of smooth and differentiable manifolds can be triangulated on a lattice of triangulable manifolds. See more comments in Sec. II. 
Proposition 5. Any $d+1 \mathrm{D}$ gauged chiral fermion theory (Model 2) that can be regularized as the low-energy effective boundary theory of a $d+2 \mathrm{D}$ gapped local lattice model in one higher dimension (Definition 3), can be regularized as the lowenergy effective theory of a local lattice model in the same $d+1 \mathrm{D}$ dimension (Definition 4), as long as the theory is free of all anomalies (given by Definition 6 and footnote 21).

We remark that for a certain anomalous $d+1 \mathrm{D}$ chiral fermion theory with an internal symmetry group $G_{f}$, their corresponding $d+2 \mathrm{D}$ topological/SPT orders may have a gapped boundary that does not break the $G_{f}$ symmetry, but has a nontrivial $G_{f}$-symmetric anomalous boundary topological order $[18,105]$ - the low-energy theory of topological order may be a $d+1 \mathrm{D} G_{f}$-symmetric topological quantum field theories (TQFT) canceling the same 't Hooft anomaly of $d+1 \mathrm{D}$ chiral fermion theory.

For such an anomalous $d+1 \mathrm{D}$ chiral fermion theory, we can have a lattice model in the same $d+1 \mathrm{D}$ dimension that exactly regularizes all the low-energy particles of the anomalous chiral fermion theories. However, the full lowenergy effective theory of the lattice model will contain an extra gauge field for a finite gauge group $G_{\text {extra }}$, prescribing the nontrivial anomalous $\mathrm{d}+1 \mathrm{D}$ topological order and TQFT. Thus, if we only concern ourselves with low-energy particles, even some anomalous gauged chiral fermion theories can be regularized by lattice models in the same dimension [23]. But the lattice models will also produce an extra $d+1 \mathrm{D} G_{\text {extra }}{ }^{-}$ gauge theory with no additional low-energy particles, but may give rise to additional extended objects such as string and brane excitations from the TQFT.

It is well known that a $\operatorname{Spin}(10)$ chiral fermion theory (Model 1) is free of all perturbative 't Hooft anomalies; similarly, it is also well known that a $\operatorname{Spin}(10)$ chiral gauge theory (Model 3) is free of all perturbative dynamical gauge anomalies [106,107]. But it is not known before whether or not the Spin(10) chiral fermion theory (Model 1) is free of all other nonperturbative global anomalies (of 't Hooft anomalies). Thus, it was also not known in the past literature whether or not the Spin(10) chiral gauge theory (Model 3) is free of all other nonperturbative global anomalies (as dynamical gauge anomalies).

Reference [23] provides an argument that the Spin(10) chiral fermion theory is free of all anomalies, by proposing a sufficient condition: A gauged chiral fermion theory in a $d+1$-dimensional spacetime with a gauge group $G_{f}$ is free of all anomalies if (0) it can be regularized as a low-energy effective boundary theory of a gapped local lattice model in one higher dimension (Definition 3), (1) there exists a nonzero Higgs field that makes all the fermions massive, and (2) $\pi_{n}\left(G_{f} / G_{\text {grnd }}\right)=0$ for $0 \leqslant n \leqslant d+2$, where $G_{\text {grnd }}$ is the unbroken gauge symmetry group for the nonzero Higgs field. The chiral fermions satisfying the above conditions can be gapped out by direct interactions or boson-induced interactions without breaking the $G_{f}$ symmetry, even when the fermion mass term is forbidden by the symmetry. This mechanism to give fermions an effective energy gap (or an effective mass) is referred to as the "mass without mass term [72]." But the above statement is based on an assumption that a smooth orientation fluctuation of Higgs field can give rise to a symmetric disordered phase. Some other related approaches have also been proposed [17,72-74,77].

In this work, we do not require the proposed conditions of Ref. [23] above, nor do we need the assumption of new fluctuating Higgs fields in Ref. [23]. Instead, we will independently and rigorously show that the above Spin(10) chiral fermion theory (Model 1) is indeed free of all 't Hooft anomalies by a cobordism group approach [Eq. (10)], and thus it can be defined on a $3+1 \mathrm{D}$ lattice; this can become a $\operatorname{Spin}(10)$ gauged chiral fermion theory (Model 2) by coupling to a Spin(10) background gauge field, or become a Spin(10) chiral gauge theory (Model 3) by dynamically gauging $\operatorname{Spin}(10)$.

\section{Gapped boundary of a state with a trivial invertible topological order with symmetry}

In the following, we show that:

(1) there exists a $3+1 \mathrm{D}$ gapped boundary for the above lattice model without breaking the Spin(10) symmetry at the low energy, and

(2) there exist nonperturbative interactions to gap the mirror world chiral fermions without breaking the Spin(10) symmetry.

The focus of this section is on showing the existence (in the mathematical sense), instead of proving the constructions (which may not be unique for the uniqueness in the mathematical sense). In Sec. III, we provide the 16 copies of the lattice model (7) that give rise to the $3+1 \mathrm{D}$ Weyl fermions in the 16-dimensional spinor representation of the $\operatorname{Spin}(10)$ on the lattice boundary; see Fig. 1.

In order to show Proposition 4, which consequently also includes Proposition 5, we break down this proposition into several related helpful subpropositions. For any well-defined $d+1 \mathrm{D}$ QFT (defined in Definition 3) that is all anomaly free (defined in Definition 6) with an internal symmetry $G_{f}$, which can live on the boundary of $\mathrm{d}+2 \mathrm{D}$ bulk regularized lattice model, we aim to show the following (in which we focus on the spatial dimension $d=3$ ):

Proposition 6. There exists a symmetric gapped boundary for the corresponding $d+2 \mathrm{D}$ bulk regularized lattice model. This $d+1 \mathrm{D}$ symmetric gapped boundary does not break any internal symmetry $G_{f}$ of the whole bulk-boundary system, and does not contribute any ground-state degeneracy (neither symmetry-breaking degeneracy nor topological degeneracy $[108,109])$.

Proposition 7. There exist nonperturbative symmetric interactions to fully gap this well-defined all-anomaly-free $d+$ 1D QFT, via deforming the QFT by adding any all-anomalyfree gapless or gapped sectors, while still preserving the full $G_{f}$ internal symmetry, without any symmetry breaking and without contributing any degeneracy (neither symmetrybreaking degeneracy nor topological degeneracy).

We will see that showing Proposition 6 is sufficient enough to show that Proposition 4 is also true. In other words, we only need Proposition 6 but do not need to prove Proposition 7, in order to prove Proposition 4.

To show Proposition 6, we first note that by a symmetric gapped boundary, we also mean that the ground-state energy $E_{0}$ (of this whole bulk-boundary system) to its higher-energy excited states (at energy $E_{1}, \ldots$, etc.) is separated by a 

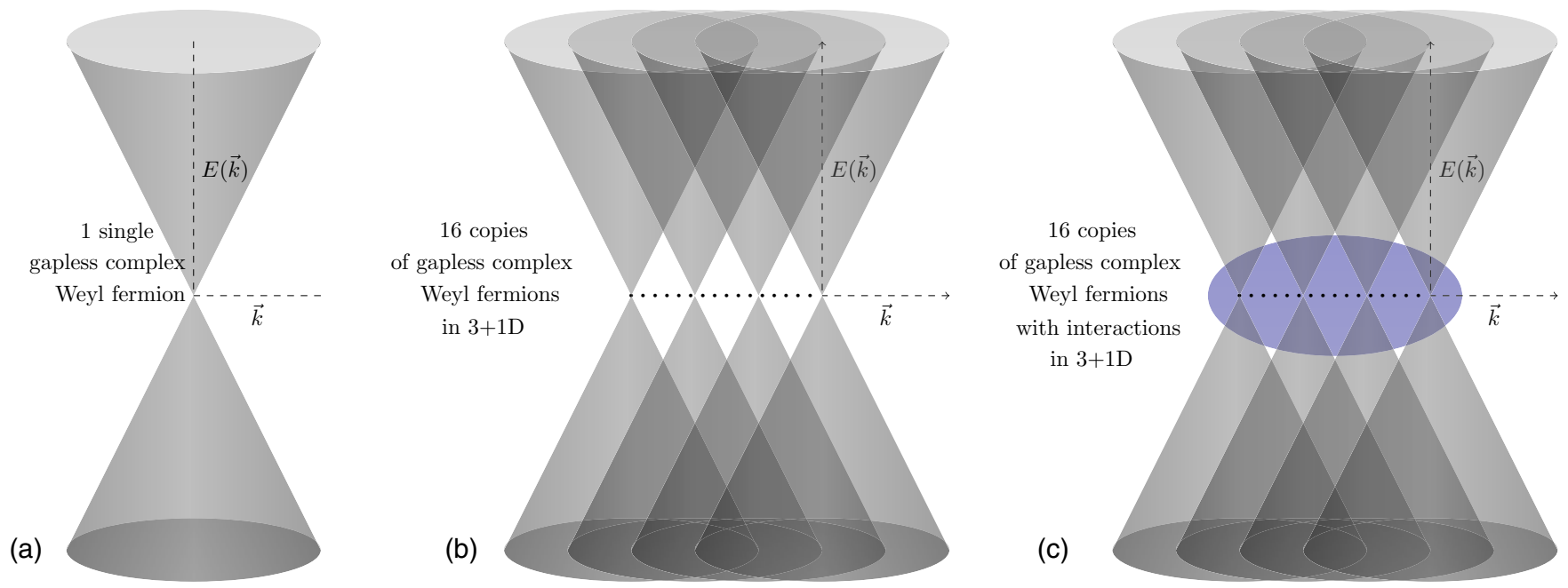

FIG. 1. (a) A lattice construction of a single Weyl fermion is given in Sec. III; the subfigure shows the gapless energy spectrum $E(\vec{k})$ of the Brillouin zone in the schematic three-dimensional momentum $\vec{k}=\left(k_{x}, k_{y}, k_{z}\right)$ space with a linear dispersion $|E(\vec{k})| \propto c|\vec{k}|$ for some effective speed of light $c$. (b) The 16 copies of the same lattice model (7) give rise to the $3+1 \mathrm{D}$ Weyl fermions at the low energy in the 16-dimensional spinor representation of the Spin(10) on the lattice boundary shown in Sec. III. The 16 gapless Weyl points (schematically the 16 dots $\bullet$ ) may be separated, but can be tuned to the same point on the $\vec{k}$-space Brillouin zone. We show that this Spin(10) chiral Weyl fermion theory is free from all 't Hooft anomalies via a cobordism theory in Sec. II. (c) There are two ways to obtain the symmetric gapped boundary for the bulk of the 16 copies of the lattice model: First, via Proposition 6, there exists a symmetric gapped boundary for the corresponding $d+2 \mathrm{D}$ bulk regularized lattice model (without the need to access from gapping out the gapless theories from interactions. Second, via Proposition 7, there exist nonperturbative symmetric interactions to fully gap this well-defined all-anomaly-free Spin(10) chiral fermion theory with 16 Weyl fermions in 16-dimensional spinor representation of the Spin(10). (Schematic interactions are drawn in the shaded blue region). In this work, we only prove Proposition 6, but we suggest some supportive evidence for Proposition 7 but without proving Proposition 7. However, applying only Proposition 6 (but without requiring Proposition 7) is sufficient enough for us to construct the Spin(10) chiral fermion theory on the lattice via Proposition 4.

finite-energy gap $\Delta_{E}=E_{1}-E_{0}>0$. Of course, by defining the energy gap $\Delta_{E}>0$ here, we should first set up a toymodel system with only such a $d+1 \mathrm{D}$ symmetric gapped boundary and a fully gapped $d+2 \mathrm{D}$ bulk. (We either have only this gapped boundary without other boundaries, or other boundaries that are also fully gapped).

If the gapped $\mathrm{d}+2 \mathrm{D}$ bulk also has a symmetric gapless boundary (say, on a $d+1 \mathrm{D}$ boundary A) other than the symmetric gapped boundary of Proposition 6 (say, on another $d+1 \mathrm{D}$ boundary B), then the gapless boundary A contributes to the low-energy spectrum at the infrared (IR) of a tiny energy subgap,

$$
\delta_{E, \mathrm{~A}} \simeq \exp (-L / \xi)
$$

which scales exponentially over the linear system size $L$ over the correlation length $\xi$; the gapped boundary B contributes to the energy spectrum only at the higher energy at a deeper ultraviolet (UV) of a finite-energy gap,

$$
\Delta_{E, \mathrm{~B}} \simeq \Delta_{E}>0,
$$

mentioned earlier. Then the whole bulk-boundary system would become gapless instead of being gapped.

The important issue is that when the $d+2 \mathrm{D}$ gapped bulk has no entanglements (i.e., no LRE nor SRE by Definition 5 ), then "the $d+1 \mathrm{D}$ symmetric gapless boundary A" and "the $d+1 \mathrm{D}$ symmetric gapped boundary B" actually cannot affect each other, and thus are isolated from each other. See more in Appendix C.
To proceed showing Proposition 6, if the bulk regularized lattice is in the gapped trivial phase (i.e., has a gapped trivial tensor product ground state), we can make a boundary by first deforming the bulk ground state (by symmetry-preserving LUT in Definition 5) into a tensor product state. Such a deformation does not close the energy gap since the bulk state is already in the symmetric gapped trivial phase. The trivial tensor product state always can have a gapped boundary with respect to a trivial vacuum ${ }^{23}$ - by saying so, we mean that we set the energy scale of the trivial vacuum (normally to below some energy scale such as a finite energy $\Delta_{E}>0$, or below an infinite energy gap $\Delta_{E} \rightarrow \infty$ ) to be the same as the energy scale of the gapped boundary (say, on B) $\Delta_{E} \simeq$ $\Delta_{E, \mathrm{~B}}>0$ in Eq. (A9). We note that the above deformation respects the on-site symmetry (if any), and the resulting tensor product state also respects the on-site symmetry. The gapped boundary does not break the on-site symmetry, and thus has no symmetry-breaking degeneracy. Since the symmetric gapped boundary has no entanglements, it therefore has no topological degeneracy (because topological degeneracy [108,109] is due to LRE defined in Definition 5).

The above completes our proof of Proposition 6.

\footnotetext{
${ }^{23}$ The trivial tensor product state always can have a gapped boundary respect to a trivial vacuum, because the trivial tensor product state is itself the same phase indistinguishable as the trivial vacuum. Thus its gapped boundary simply is the trivial gapped domain wall between the same phase [110,111].
} 
In Appendix D, we can also show Proposition 6 by a second viewpoint: a derivation from the classification of quantum phases of matter and their phase transitions.

This second viewpoint from the classification of quantum phases of matter shows that there is no need for an energy-gap closing phase transition. By maintaining a finite-energy gap $\Delta_{E}$ between two phases, there must exist a symmetric gapped boundary between two phases, and thus we have given an alternative proof of Proposition 6.

The slight conceptual difference between the first viewpoint and the second viewpoint is that the first is about the one-spatial-dimensional-lower phase boundary in $d+1 \mathrm{D}$ between two $d+2 \mathrm{D}$ phases, while the second is about no need for the phase transition in $d+2 \mathrm{D}$ between two $d+2 \mathrm{D}$ phases in a quantum phase diagram (at zero temperature $T=0$ ) by tuning a certain coupling $g$.

To show Proposition 4, we consider a $d+2 \mathrm{D}$ bulk regularized lattice model that regularizes the $d+1 \mathrm{D}$ QFT as its boundary theory by Definition 3 . We choose the bulk lattice model to be a slab of finite thickness, such that one boundary of the slab regularizes the QFT (Boundary A), and the other boundary is a symmetric gapped boundary (Boundary B) in Proposition 6. We apply Proposition 6 that was proven earlier. Here a slab of finite thickness is always achievable for this system (especially for the gauged chiral fermion problem of Model 1 and Model 2) because of the isolation between two $d+1 \mathrm{D}$ boundaries A and B due to Appendix C's remark (i) on the isolation of the energy scale and remark (ii) on the isolation of the mutual entanglement; see Appendix $\mathrm{C}$ for the energy scale and mutual entanglement between gapless and gapped boundaries.

Thus the low-energy physics of the $d+2 \mathrm{D}$ slab is described by this $d+1 \mathrm{D}$ QFT. A lattice model of this $d+2 \mathrm{D}$ slab of a finite thickness can be constructed explicitly as a lattice model in one lower dimension $(d+1 \mathrm{D})$, by rewriting the "quantum Hilbert space associated with different lattice sites along the finite width thickness w (i.e., an extra small dimension along w)" to "quantum Hilbert space associated with finite orbitals per site" in $d+1 \mathrm{D}$.

This completes our proof of Proposition $4 .^{24}$

\section{A deformation class of all-anomaly-free well-defined QFTs}

For Proposition 7, we again consider a bulk lattice model that regularizes the QFT as its boundary theory by Definition 3. Since the same bulk model can also have a symmetric gapped boundary according to Proposition 6, we thus ask: How can we modify the symmetric interactions in the QFT to make it into a fully symmetric gapped theory describing the symmetric gapped boundary?

One key ingredient is that there are more degrees of freedom given by the full Hilbert space to help us reach the goal. We may be able to access the symmetric gapped phase not only within this specific well-defined all-anomaly-free QFT, but also a higher energy spectrum by engineering all

\footnotetext{
${ }^{24}$ As we said earlier, we do not need Proposition 7 to show Proposition 4.
}

possible degrees of freedom and their symmetry-preserving local interactions.

We do not have a direct proof of Proposition 7, but we have several supportive pieces of evidence to argue that Proposition 7 should be true:

(a) "The deformation classes of QFTs" advocated by Seiberg [112]: Given a continuum QFT with some energy scale $\Lambda$, with a given global symmetry and derivable 't Hooft anomaly of global symmetry. We are allowed to add arbitrary degrees of freedom and new fields preserving the symmetries (and selection rules) and with no additional anomaly (without modifying the original 't Hooft anomaly) at some energies. The new degrees of freedom do not directly affect the dynamics at lower energies. Next, we can deform the parameters of this larger theory with the new degrees of freedom, by making the new degrees of freedom interacting with the original QFT, which do affect the dynamics. This is a much larger space of theories, which can land into different new phases with different dynamics. Seiberg names all these possible deformations of QFT as a deformation class of the QFT.

Seiberg [112] conjectured that given two QFTs, say partition functions $\mathbf{Z}_{1}$ and $\mathbf{Z}_{2}$, in the same spacetime dimension with:

(i) the same global symmetry (and selection rules), and

(ii) the same 't Hooft anomalies,

we can always add new degrees of freedom at short distances so that we can interpolate between two QFTs: The two QFTs, $\mathbf{Z}_{1}$ and $\mathbf{Z}_{2}$, are in the same deformation class of QFT. In other words, this also means that the deformation class of QFT can be determined and defined by the symmetries and the 't Hooft anomalies of QFT. Seiberg's conjecture is in fact shown to be true for many examples.

What we claim on Proposition 7 is indeed a special case of Seiberg's proposal [112]: We consider the deformation class of the anomaly-free well-defined QFT, containing the trivial gapped phase [e.g., a symmetric gapped Spin(10) boundary] and a gapless phase [e.g., a symmetric gapless Spin(10) chiral fermion theory], in which both have (i) the same global symmetry (and selection rules) and (ii) no 't Hooft anomalies (that we will show via a cobordism theory in Sec. II). If Seiberg's proposal [112] is true, our proposal must also be true.

(b) We can start from the $d+1 \mathrm{D}$ symmetric gapless all-anomaly-free theory, and adding new $d+1 \mathrm{D}$ symmetric gapped all-anomaly-free sectors (this is analogous to Seiberg's proposal [112]).

Moreover, we can also add additional gapless all-anomalyfree sectors in the various possible representations (Rep) of symmetry. ${ }^{25}$ The symmetry organizes the (both gapped and gapless) energy eigenstates in the energy spectrum into various possible representations of the symmetry group, whose

\footnotetext{
${ }^{25}$ For example, the trivial Rep of pairs of left- and right-moving $3+1 \mathrm{D}$ Weyl fermions $\psi_{L}$ and $\psi_{R}$ in the trivial Rep of Spin(10). Then, adding their mass term, e.g., $m\left(\psi_{L}^{\dagger} \psi_{R}+\psi_{R}^{\dagger} \psi_{L}\right)$ (only for these additional trivial Rep gapless sectors), does not break the Spin(10) symmetry.
} 
selection rules constrain the interactions and dynamics between states in the energy spectrum.

Based on (a) and (b), we propose that Proposition 7 is also true.

\section{APPENDIX B: CONSTRUCT A LOCAL BOSONIC LATTICE MODEL REALIZING A 3 + 1D Spin(10) GAUGED CHIRAL FERMION THEORY}

Below we will use the slave-particle/parton approach [78-80] to explicitly construct a $4+1 \mathrm{D}$ local bosonic lattice model, whose boundary can give rise to the dynamical Spin(10) chiral gauge theory coupled to Weyl fermions (Model 3) in the 16-dimensional representation. We start with a fermionic model on a 4D cubic lattice. On each site, we have $16 N_{f}$ complex fermions $\hat{\psi}_{\alpha, m}$, with $\alpha=1, \ldots, 16$, and $m=1, \ldots, N_{f}{ }^{26}$ So on each side, there are $2^{16 N_{f}}$ states. Now we project into the even fermion subspace on each site, and turn the fermionic model into a bosonic model with $2^{16 N_{f}-1}$ states per site. The Hamiltonian for such a bosonic model is given by

$$
\begin{aligned}
\hat{H}_{1} & =\sum_{\langle i j\rangle} \sum_{\alpha \beta}\left(\hat{\chi}_{i j}^{\alpha \beta}\right)^{\dagger} \hat{\chi}_{i j}^{\alpha \beta}+\sum_{i}(-)^{i} \hat{n}_{i}, \\
\hat{\chi}_{i j}^{\alpha \beta} & =\sum_{m} \hat{\psi}_{\alpha, m, i}^{\dagger} \hat{\psi}_{\beta, m, j}, \quad \hat{n}_{i}=\sum_{m, \alpha} \hat{\psi}_{\alpha, m, i}^{\dagger} \hat{\psi}_{\alpha, m, i} .
\end{aligned}
$$

The above model has $[\mathrm{U}(16)]^{N_{\text {site }}}$ local symmetry. In the large $N_{f}$ limit, $\hat{\chi}_{i j}^{\alpha \beta}$ is weakly fluctuating and can be replaced by $\chi\left(e^{i A_{i j}}\right)_{\alpha \beta}=\left\langle\hat{\chi}_{i j}^{\alpha \beta}\right\rangle$ expectation value and $A_{i j}$ is a $16 \times 16$ Hermitian matrix to describe the U(16) gauge fluctuation. This leads to the following emergent U(16) gauge theory (at a mean-field level):

$$
\begin{aligned}
\hat{H}_{1}^{\text {mean }}= & \sum_{\langle i j\rangle} \sum_{\alpha \beta, m}\left[\hat{\psi}_{\alpha, m, i}^{\dagger} \chi^{*}\left(e^{-i A_{i j}}\right)_{\alpha \beta} \hat{\psi}_{\beta, m, j}+\text { H.c. }\right] \\
& +\sum_{i}(-)^{i} \hat{n}_{i} .
\end{aligned}
$$

The ground state is given by $A_{i j}=0$. The emergent fermions are in a fully gapped product state and the bosonic model $\hat{H}_{1}$ gives rise to a U(16) gauge theory at low energies.

Next, we reduce the U(16) gauge theory to the Spin(10) gauge theory by adding a term,

$$
\hat{H}_{2}=\sum_{i, m} \Gamma^{\alpha \beta \gamma \lambda} \hat{\psi}_{\alpha, m, i} \hat{\psi}_{\beta, m, i} \hat{\psi}_{\gamma, m, i} \hat{\psi}_{\lambda, m, i}+\text { H.c. }
$$

to break the $[\mathrm{U}(16)]^{N_{\text {site }}}$ local symmetry to a $[\operatorname{Spin}(10)]^{N_{\text {site }}}$ local symmetry where the fermions $\psi_{\alpha, m, i}$ form the 16dimensional spinor representation. Here, $\Gamma^{\alpha \beta \gamma \lambda}$ is the antisymmetric tensor which is invariant under the Spin(10) transformations. The $4+1 \mathrm{D}$ bosonic model $\hat{H}_{1}+\hat{H}_{2}$ will give rise to an emergent Spin(10) gauge theory with the fermions in a fully gapped product state. Those fermions are also gapped on the boundary.

\footnotetext{
${ }^{26} \mathrm{We}$ introduce a new flavor parameter $N_{f}$, so that we gain a benefit to do a large $N_{f}$ analysis for $N_{f} \gg 1$ or further, $N_{f} \rightarrow \infty$.
}

To have gapless Weyl fermions on the boundary, we add the third term,

$$
\hat{H}_{3}=\sum_{i j}\left(t_{i j}^{a b} \hat{c}_{\alpha, a, i}^{\dagger} \hat{c}_{\beta, b, j} \hat{\chi}_{i j}^{\alpha \beta}+\text { H.c. }\right) .
$$

Now, on each site, we have fermions $\hat{\psi}_{\alpha, m}$ and $\hat{c}_{\alpha, a}$, but we still project into the subspace with an even fermion per site. The $\hat{H}_{1}+\hat{H}_{2}+\hat{H}_{3}$ acts within this subspace. So the model is still a lattice bosonic model. When $t_{i j}$ is given by Eq. (7), the model

$$
\hat{H}_{1}+\hat{H}_{2}+\hat{H}_{3}
$$

will give rise to emergent massless Weyl fermions on the boundary coupled to the Spin(10) gauge field.

Consider a $4+1 \mathrm{D}$ slab of the local bosonic lattice model described by $\hat{H}_{1}+\hat{H}_{2}+\hat{H}_{3}$. In the main text, based on the complete classification of the 't Hooft anomaly of the group $G=[\operatorname{Spin}(5) \times \operatorname{Spin}(10)] / \mathbb{Z}_{2}^{f}$ [in Eq. (10) and Eq. (14)], we have shown that there exists a symmetric gapped boundary by Propositions 4 and 5 (or there exists a symmetric boundary gapping interaction, called $\hat{H}_{\text {int.bdry.nonpert }}$ ), which allows us to gap out the boundary Weyl fermions (without inducing a boundary $3+1 \mathrm{D}$ topological order [18]) on one of the surfaces of the slab.

In this case, by including such interactions $\hat{H}_{\text {int.bdry.nonpert }}$ into (B5), we propose a Hamiltonian

$$
\hat{H}_{1}+\hat{H}_{2}+\hat{H}_{3}+\hat{H}_{\text {int.bdry.nonpert }} \text {. }
$$

Then by applying Eq. (18)'s finite-width/layer lattice dimensional reduction, the $4+1 \mathrm{D}$ slab, with a finite width in the extra dimension, indeed becomes a $3+1 \mathrm{D}$ local bosonic lattice model that regularizes a $3+1 \mathrm{D}$ dynamical $\operatorname{Spin}(10)$ gauge theory coupled to Weyl fermions in the 16-dimensional spinor representation.

\section{APPENDIX C: ENERGY SCALE AND MUTUAL ENTANGLEMENT BETWEEN GAPLESS AND GAPPED BOUNDARIES}

The important issue is that when the $d+2 \mathrm{D}$ gapped bulk has no entanglements (i.e., no LRE or SRE by Definition 5), "the $d+1 \mathrm{D}$ symmetric gapless boundary A" and "the $d+1 \mathrm{D}$ symmetric gapped boundary B" actually cannot affect each other, and thus are isolated from each other, in the following sense:

(i) Energy scale. Boundary A and Boundary B are decoupled below the energy scale $\simeq \Delta_{E, \mathrm{~B}}$. But when the energy is above the scale $\gtrsim \Delta_{E, \mathrm{~B}}$, the energy spectra of $\mathrm{A}, \mathrm{B}$, and the bulk may affect and mix together with each other.

(ii) Mutual entanglement. Although the $d+1 \mathrm{D}$ symmetric gapless boundary A is highly entangled (due to the low-lying massless chiral fermions as the energy gapless spectrum), and the symmetric gapped boundary B is trivially gapped with no entanglements as a tensor product state on Boundary B, thanks to the trivial gapped bulk, Boundary A and Boundary $\mathrm{B}$ on two sides have no entanglements in between. More precisely, if we choose a $d+1 \mathrm{D}$ bipartite cut inside the $d+2 \mathrm{D}$ gapped bulk, we get a zero bipartite Von Neumann 
entanglement entropy,

$$
\begin{aligned}
S_{\mathrm{EE}} & =S\left(\rho_{A}\right)=-\operatorname{Tr}\left[\rho_{A} \ln \rho_{A}\right] \\
& =-\operatorname{Tr}\left[\rho_{B} \ln \rho_{B}\right]=S\left(\rho_{B}\right)=0,
\end{aligned}
$$

for the mutual entanglement between two sides. (This understanding is consistent with the entanglement structure discussed in Definition 5).

For the lattice regularization of the gauged chiral fermion problem, we should emphasize that our statements in (i) and (ii) apply to Model 1 (a chiral fermion theory) and Model 2 (a gauged chiral fermion theory). However, we do not intend to apply our statements in (i) and (ii) to Model 3 (a chiral gauge theory) — once we dynamically gauge the internal global symmetry for the bulk-boundary coupled system, then "the bulk, Boundary A and Boundary B" form altogether highly entangled quantum states (as a dynamical gauge theory). The $S_{\mathrm{EE}}$ of the dynamically gauged system (Model 3), based on the previous bipartite cut in Eq. (C1), is generically nonzero.

\section{APPENDIX D: THE EXISTENCE OF SYMMETRIC GAPPED BOUNDARY VIA QUANTUM PHASE TRANSITIONS}

A derivation can also be obtained from the classification of quantum phases of matter and their phase transitions. To give a proof of Proposition 6, all we need to show is that there exists a LUT deformation path (Definition 5) between two bulk gapped quantum phases:

(1) Bulk phase: The $d+2 \mathrm{D}$ bulk regularized lattice model which has a symmetric gapped trivial tensor product ground state, with a finite energy gap $\Delta_{E}$

(2) Trivial gapped vacuum phase (mentioned above),

such that this LUT deformation path satisfies the following criteria:

(a) it does not close the energy gaps between the bulk phase and the trivial vacuum phase [i.e., no gap closing, and thus no gapless modes and no zero-mode degeneracy (=ground-state degeneracy)].

(b) it does not break the internal global symmetry given by the bulk phase (i.e., $G_{f}=\operatorname{Spin}(10)$ for the $\operatorname{Spin}(10)$ gauged chiral fermion problem).

This LUT deformation path can be regarded as a path labeled by $g$ in the quantum phase diagram (at zero temperature, $T=0$ ) by tuning a parameter (i.e., a coupling constant) $g$ of the lattice Hamiltonian $\hat{H}(g)$ such that the ground state $\left|\Psi_{\text {g.s. }}(g)\right\rangle$ is unitarily evolving under this LUT along the deformation path. Then we can prove the claim of (a) and (b), either by "proof by a contradiction" or by directly "constructing such a LUT deformation path."

"A proof by a contradiction." Suppose, given by engineering arbitrary symmetry-preserving (e.g., $G_{f}$ ) local interactions for the lattice Hamiltonian, such a path in the phase diagram is still impossible between two phases (the bulk phase and the trivial gapped vacuum phase). Then there must be a phase transition between two phases, and the two phases should be different quantum phases-in fact, they should be different SPT phases within the $G_{f}$ symmetry. But as we emphasize that both phases have symmetric gapped trivial tensor product ground states, they must be in the same trivial SPT phase, and thus the same trivial gapped vacuum, at least below the energy gap $\Delta_{E}$ of the bulk phase. This leads to a contradiction, and thus we end the proof successfully.

"Constructing such a LUT deformation path." This path construction is basically what we had in the earlier proof. Since both phases are symmetric gapped trivial phases (both a trivial SPT phase and a trivial gapped vacuum respect to the $G_{f}$ symmetry), the LUT deformation path is simply the deformation to make both symmetric gapped trivial phases become exactly the same symmetric gapped trivial tensor product states in a certain "canonical basis" with respect to the $G_{f}$ symmetry. (Normally, it is known as the symmetric disordered phase, where the canonical basis is chosen to be the dual variable of the symmetry-breaking basis).

\section{APPENDIX E: COBORDISM THEORY AND CLASSIFICATION OF ALL POSSIBLE INVERTIBLE ANOMALIES RELATED TO SU(5) AND SO(10) GRAND UNIFICATIONS}

Here we provide the cobordism group calculations classifying all potential invertible 't Hooft anomalies of $\mathrm{SU}(5)$, Spin(10), and Spin(18) chiral fermion theories. Our calculations are crucial for showing all gauge anomaly-free conditions for $\mathrm{SU}(5), \mathrm{SO}(10)$, and $\mathrm{SO}(18)$ grand unifications. Notice that other related work [66] computes $\Omega_{\mathrm{D}}^{\mathrm{Spin} \times \mathrm{SU}(5)}$ and $\Omega_{\mathrm{D}}^{\mathrm{Spin} \times \operatorname{Spin}(10)}$ based on a different method, i.e., AtiyahHirzebruch spectral sequence (AHSS), while our work focus on $\Omega_{\mathrm{D}}^{\mathrm{Spin} \times \mathrm{SU}(5)}$ and $\Omega_{\mathrm{D}}^{\mathrm{Spin} \times \operatorname{Spin}(10) / \mathbb{Z}_{2}^{f}}$, also based on a more powerful Adams spectral sequence. See, also, Ref. [55].

\section{Adams spectral sequence}

The Adams spectral sequence shows the following:

$$
\operatorname{Ext}_{\mathcal{A}_{p}}^{s, t}\left(\mathrm{H}^{*}\left(Y, \mathbb{Z}_{p}\right), \mathbb{Z}_{p}\right) \Rightarrow \pi_{t-s}(Y)_{p}^{\wedge},
$$

where Ext denotes the extension functor, $\mathcal{A}_{p}$ is the $\bmod \mathrm{p}$ Steenrod algebra, and $Y$ is any spectrum. The $\mathrm{H}^{*}\left(Y, \mathbb{Z}_{p}\right)$ is an $\mathcal{A}_{p}$-module whose internal degree $t$ is given by the $*$. The $\pi_{t-s}(Y)_{p}^{\wedge}$ is the $p$-completion of the $(t-s)$-th homotopy group of the spectrum $Y$. We note that for any finitely generated Abelian group $\mathcal{G}, \mathcal{G}_{p}^{\wedge}=\lim _{n \rightarrow \infty} \mathcal{G} / p^{n} \mathcal{G}$ is the $p$ completion of $\mathcal{G}$; if $\mathcal{G}$ contains an infinite group $\mathbb{Z}$, then $\mathcal{G}_{p}^{\wedge}$ is the ring of $p$-adic integers. Here, the $\mathcal{G}$ is meant to be substituted by a homotopy group $\pi_{t-s}(Y)_{p}^{\wedge}$ in (E2). Here are some explanations and inputs:

(1) Here the double-arrow " $\Rightarrow$ " means "convergent to." The $E_{2}$ page contains groups $\mathrm{Ext}^{s, t}$ with double indices $(s, t)$, and we reindex the bidegree by $(t-s, s)$. There are differentials $d_{2}$ in the $E_{2}$ page, which are arrows from $(t-$ $s, s)$ to $(t-s-1, s+2)$. That is, $\mathrm{Ext}^{s, t} \rightarrow \mathrm{Ext}^{s+2, t+1}$. Take $\operatorname{Ker} d_{2} / \operatorname{Im} d_{2}$ at each $(t-s, s)$; then we get the $E_{3}$ page. Repeat this procedure, and we get the $E_{4}$ page, $E_{5}$ page, and so on. Finally, the $E_{r}$ page equals the $E_{r+1}$ page (there are no differentials) for $r \geqslant N$; we call this $E_{N}$ page as the $E_{\infty}$ page, and we can read the result $\pi_{D}$ at $D=t-s$. See further details discussed in Ref. [30], Sec. 2.3. 
(2) In Adams spectral sequence, we consider $\operatorname{Ext}_{R}^{s, t}\left(L, \mathbb{Z}_{p}\right)$. Here we have the ring or the algebra $R=\mathcal{A}_{p}$ or $\mathcal{A}_{2}(1)$ for $p=2$, and the $L$ is a $R$-module. The $\mathcal{A}_{2}(1)$ is the subalgebra of $\mathcal{A}_{2}$ generated by the Steenrod square $\mathrm{Sq}^{1}$ and $\mathrm{Sq}^{2}$. The index $s$ refers to the degree of resolution, and the index $t$ is the internal degree of the $R$-module $L$. Ext groups are defined by first taking a projective $R$-resolution $P_{\bullet}$ of $L$, then, second, computing the (co)homology group of the (co)chain complex $\operatorname{Hom}\left(P_{\bullet}, \mathbb{Z}_{p}\right)$. A $P_{\bullet}$ is a resolution, which is an exact sequence of modules. Here a projective $R$-resolution $P_{\boldsymbol{\bullet}}$ is an exact sequence of $R$-modules $\cdots \rightarrow P_{s} \rightarrow P_{s-1} \rightarrow \cdots \rightarrow P_{0} \rightarrow L$, where $P_{s}$ is projective for $s \geqslant 0$.

\section{Thom-Madsen-Tillmann spectrum and Pontryagin-Thom isomorphism}

For $Y=M T G$, where $M T G$ is the Thom-MadsenTillmann spectrum $M T G$ of a group $G$, the Adams spectral sequence shows the following:

$$
\begin{aligned}
& \operatorname{Ext}_{\mathcal{A}_{p}}^{s, t}\left(\mathrm{H}^{*}\left(M T G, \mathbb{Z}_{p}\right), \mathbb{Z}_{p}\right) \\
& \quad \Rightarrow \pi_{t-s}(M T G)_{p}^{\wedge}=\left(\Omega_{D=t-s}^{G}\right)_{p}^{\wedge} .
\end{aligned}
$$

The last equality is that by the generalized Pontryagin-Thom isomorphism, we have an equality between the $D$-th bordism group of $G$ given by $\Omega_{D}^{G}$ and the $D$-th homotopy group of $M T G$ given by $\pi_{D}(M T G)$, namely,

$$
\Omega_{D}^{G}=\pi_{D}(M T G) .
$$

We also compute the cobordism group of topological phases (TP) defined in [29] as

$$
\operatorname{TP}_{D}(G) .
$$

The $\operatorname{TP}_{D}(G)$ classifies deformation classes of reflection positive invertible $d$-dimensional extended topological field theories with symmetry group $G_{D}$. The $\operatorname{TP}_{D}(G)$ and the bordism group $\Omega_{D}^{G}$ are related by a short exact sequence,

$$
0 \rightarrow \operatorname{Ext}^{1}\left(\Omega_{D}^{G}, \mathbb{Z}\right) \rightarrow \operatorname{TP}_{D}(G) \rightarrow \operatorname{Hom}\left(\Omega_{D+1}^{G}, \mathbb{Z}\right) \rightarrow 0 .
$$

We can compute the $E_{2}$ page of the $\mathcal{A}_{2}(1)$-module based on Lemma 11 of [30]. More precisely, in order to compute $\operatorname{Ext}_{\mathcal{A}_{2(1)}}^{s, t}\left(L_{2}, \mathbb{Z}_{2}\right)$, we find a short exact sequence of $\mathcal{A}_{2}(1)$ modules,

$$
0 \rightarrow L_{1} \rightarrow L_{2} \rightarrow L_{3} \rightarrow 0,
$$

then we apply Lemma 11 of [30] to compute $\operatorname{Ext}_{\mathcal{A}_{2(1)}}^{s, t}\left(L_{2}, \mathbb{Z}_{2}\right)$ by the given data of $\operatorname{Ext}_{\mathcal{A}_{2}(1)}^{s, t}\left(L_{1}, \mathbb{Z}_{2}\right)$ and $\operatorname{Ext}_{\mathcal{A}_{2}(1)}^{s, t}\left(L_{3}, \mathbb{Z}_{2}\right)$. Our strategy is choosing $L_{1}$ to be the direct sum of suspensions of $\mathbb{Z}_{2}$ on which $\mathrm{Sq}^{1}$ and $\mathrm{Sq}^{2}$ act trivially; then we take $L_{3}$ to be the quotient of $L_{2}$ by $L_{1}$. We can use this procedure again and again until $\operatorname{Ext}_{\mathcal{A}_{2(1)}}^{s, t}\left(L_{3}, \mathbb{Z}_{2}\right)$ is determined.

If $G=\operatorname{Spin} \times G^{\prime}$, then $\mathrm{B} G=\mathrm{B}\left(\operatorname{Spin} \times G^{\prime}\right)=\mathrm{BSpin} \times$ $\mathrm{B} G^{\prime}$. By definition, the Madsen-Tillmann spectrum $M T G=$ Thom $(\mathrm{B} G,-V)$ where $V$ is the induced virtual bundle of dimension 0 by the map $\mathrm{B} G \rightarrow \mathrm{BO}$. By the properties of Thom space (see the discussions in Ref. [30], Sec. 1.3), we have

$$
M T\left(\operatorname{Spin} \times G^{\prime}\right)=M \operatorname{Spin} \wedge\left(\mathrm{B} G^{\prime}\right)_{+} .
$$

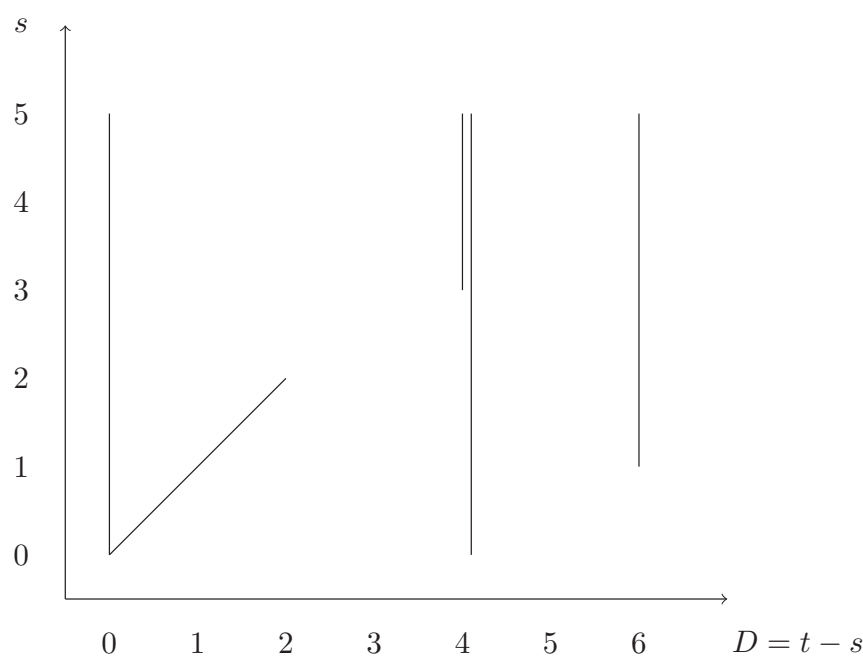

FIG. 2. Adams chart for $\Omega_{D}^{\mathrm{Spin} \times \mathrm{SU}(5)}$.

The $\wedge$ is the smash product.

Below we will use (E2) and (E3) to compute the $D$-th bordism group of $G$ given by $\Omega_{D}^{G}$. Then we will use the (E5) and the techniques around (E6) to compute the $D$-th cobordism group of topological phases of $G$ given by $\operatorname{TP}_{D}(G)$.

\section{Cobordism groups and topological phases for Spin $\times \mathrm{SU}(5)$ : SU(5) grand unification}

We consider $G=\operatorname{Spin} \times \mathrm{SU}(5)$ for the Georgi-Glashow SU(5) grand unification [5]; the Thom-Madsen-Tillmann spectrum $M T G$ of the group $G$ is

$$
M T G=M \operatorname{Spin} \wedge(\mathrm{BSU}(5))_{+} .
$$

The $T$ in $M T G$ means the $G$-structures are on tangent bundles instead of normal bundles. For Spin, the Thom-MadsenTillmann spectrum $M T$ Spin $=M$ Spin is equivalent to the Thom spectrum, which splits $M$ Spin $=k o \vee \Sigma^{8} k o \vee \cdots$. The $k o$ is the (-1)-connected cover of the real $K$-theory spectrum. The $\wedge$ is the smash product and the $\vee$ is the wedge sum. The $(\mathrm{BSU}(5))_{+}$is the disjoint union of the classifying space BSU(5) and a point. ${ }^{27}$

For the dimension $D=t-s<8$, since there is no odd torsion, ${ }^{28}$ by $M T G=M$ Spin $\wedge X$, then the $D$-th homotopy group $\pi_{D}(M T G)=\pi_{D}(k o \wedge X)$ for $D<8$. So, for the dimension $D=t-s<8$, we have

$$
\operatorname{Ext}_{\mathcal{A}_{2}(1)}^{s, t}\left(\mathrm{H}^{*}\left(X, \mathbb{Z}_{2}\right), \mathbb{Z}_{2}\right) \Rightarrow\left(\Omega_{D=t-s}^{G}\right)_{2}^{\wedge} .
$$

Hence, for $M T G=M \operatorname{Spin} \wedge(\mathrm{BSU}(5))_{+}$, for the dimension $D=t-s<8$, by (E9), we have the Adams spectral sequence,

$$
\operatorname{Ext}_{\mathcal{A}_{2}(1)}^{s, t}\left(\mathrm{H}^{*}\left(\mathrm{BSU}(5), \mathbb{Z}_{2}\right), \mathbb{Z}_{2}\right) \Rightarrow \Omega_{t-s}^{\mathrm{Spin} \times \mathrm{SU}(5)} .
$$

\footnotetext{
${ }^{27}$ For a topological space $X$, it is a standard convention to denote $X_{+}$as the disjoint union of $X$ and a point. Note that the reduced cohomology of $X_{+}$is exactly the ordinary cohomology of $X$.

${ }^{28} \mathrm{By}$ computation using the mod $p$ Adams spectral sequence for an odd prime $p$, we find there is no odd torsion.
} 
TABLE I. Bordism group $\Omega_{D}^{\operatorname{Spin} \times \operatorname{SU}(5)} \cdot \sigma$ is the signature of manifold. The $c_{j}$ is the $j$ th Chern class of the associated vector bundle of $\mathrm{SU}(n)$. Note that $c_{3}=\mathrm{Sq}^{2} c_{2}=\left(w_{2}+w_{1}^{2}\right) c_{2}=0 \bmod 2$ on Spin 6-manifolds. Actually, $\Omega_{D}^{\operatorname{Spin} \times \operatorname{SU}(n)}=\Omega_{D}^{\operatorname{Spin} \times \operatorname{SU}(n+1)}$ for $n \geqslant 3$ and $0 \leqslant D \leqslant 6$. See, also, Ref. [55].

\begin{tabular}{lcc}
\hline \hline \multicolumn{3}{c}{ Bordism group } \\
\hline$D$ & $\Omega_{D}^{\text {Spin } \times \text { SU }(5)}$ & Generators \\
\hline 4 & $\mathbb{Z}^{2}$ & $\frac{\sigma}{16}, c_{2}$ \\
5 & 0 & $\frac{c_{3}}{2}$ \\
6 & $\mathbb{Z}$ & \\
\hline \hline
\end{tabular}

The $\mathcal{A}_{2}(1)$-module structure of $\mathrm{H}^{*}\left(\mathrm{BSU}(5), \mathbb{Z}_{2}\right)$ below degree 6 is shown in Ref. [55], Sec. 6, Fig. 29, and the $E_{2}$ page is shown in Fig. 2. Here we have used the correspondence between the $\mathcal{A}_{2}(1)$-module structure and the $E_{2}$ page shown in Appendix A of Ref. [55].

In the Adams chart, the horizontal axis labels the integer degree $D=t-s$ and the vertical axis labels the integer degree $s$. The differential $d_{r}^{s, t}: E_{r}^{s, t} \rightarrow E_{r}^{s+r, t+r-1}$ is an arrow starting at the bidegree $(t-s, s)$ with direction $(-1, r) . E_{r+1}^{s, t}:=\frac{\operatorname{Ker} d_{r}^{s, t}}{\operatorname{Im} d_{r}^{s-r, t-r+1}}$ for $r \geqslant 2$. There exists $N$ such that $E_{N+k}=E_{N}$ stabilized for $k>0$; we denote the stabilized page $E_{\infty}:=E_{N}$.

To read the result from the Adams chart in Fig. 2, we look at the stabilized $E_{\infty}$ page, where one dot indicates a finite group $\mathbb{Z}_{p}$, and a vertical finite line segment connecting $n$ dots indicates a finite group $\mathbb{Z}_{p^{n}}$. But when $n=\infty$, the infinite line connecting infinite dots indicates an infinite group, an integer $\mathbb{Z}$. Here, $p$ is given by the $\bmod p$ Steenrod algebra $\mathcal{A}_{p}$ in (E2). Here, in Fig. 2, $p=2$, and we can read from the Adams chart $\Omega_{0}^{\mathrm{Spin} \times \mathrm{SU}(5)}=\mathbb{Z}$ (an infinite line), $\Omega_{1}^{\mathrm{Spin} \times \mathrm{SU}(5)}=$ $\mathbb{Z}_{2}$ (a dot), $\Omega_{2}^{\text {Spin } \times S U(5)}=\mathbb{Z}_{2}$ (a dot), $\Omega_{3}^{\text {Spin } \times S U(5)}=0$ (nothing), $\Omega_{4}^{\mathrm{Spin} \times \mathrm{SU}(5)}=\mathbb{Z}^{2}$ (two infinite lines), $\Omega_{5}^{\mathrm{Spin} \times \mathrm{SU}(5)}=0$ (nothing), and $\Omega_{6}^{\mathrm{Spin} \times \mathrm{SU}(5)}=0$ (an infinite line).

\section{Classification of all invertible anomalies of Spin $\times$ SU(5) fermion theories}

By (E2) and (E3), we obtain the bordism group $\Omega_{D}^{\mathrm{Spin} \times \mathrm{SU}(5)}$ shown in Table I, focusing on $D=4,5,6$.

By (E5) and (E6), we obtain the cobordism group $\mathrm{TP}_{D}($ Spin $\times \mathrm{SU}(5))$ shown in Table II, focusing on $D=4,5$.

TABLE II. Topological phase classification (三 TP) as a cobordism group $\mathrm{TP}_{D}(\operatorname{Spin} \times \mathrm{SU}(5))$, following Table I. See, also, Ref. [55].

\begin{tabular}{lcc}
\hline \hline \multicolumn{3}{c}{ Cobordism group } \\
\hline$d$ & $\mathrm{TP}_{D}(\mathrm{Spin} \times \mathrm{SU}(5))$ & Generators \\
\hline 4 & 0 & \\
5 & $\mathbb{Z}$ & $\frac{1}{2} \mathrm{CS}_{5}^{\mathrm{SU}(5)}$ \\
\hline \hline
\end{tabular}

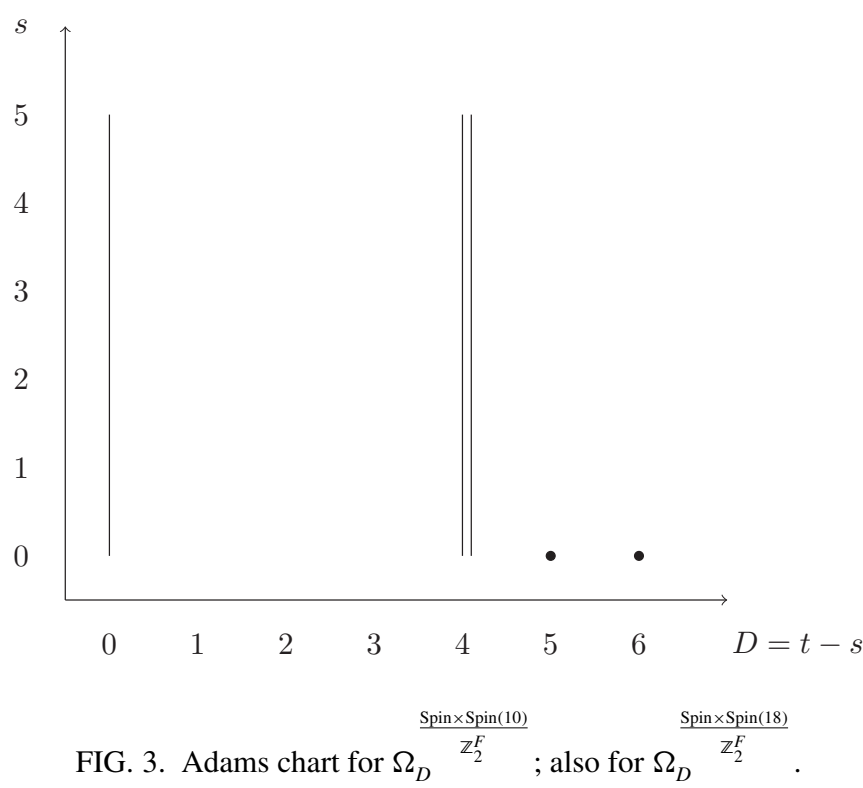

4. Cobordism groups and topological phases for $\frac{\operatorname{Spin} \times \operatorname{Spin}(10)}{\mathbb{Z}_{2}^{f}}$ and $\frac{\operatorname{Spin} \times \operatorname{Spin}(18)}{\mathbb{Z}_{2}^{f}}: \operatorname{SO}(10)$ and $\operatorname{SO}(18)$ grand unification

We consider $G=\frac{\operatorname{Spin} \times \operatorname{Spin}(10)}{\mathbb{Z}_{2}^{F}}$ for the Fritzsch-Minkowski $\mathrm{SO}(10)$ grand unification [6]; the Thom-Madsen-Tillmann spectrum $M T G$ of the group $G$ is

$$
M T G=M \operatorname{Spin} \wedge \Sigma^{-10} M \operatorname{SO}(10) .
$$

The $T$ in $M T G$ means the $G$-structures are on tangent bundles instead of normal bundles. In this case, we have $w_{2}(T M)=$ $w_{2}\left(V_{\mathrm{SO}(10)}\right)$.

For the dimension $D=t-s<8$, since there is no odd torsion (see footnote 28), by $M T G=M \operatorname{Spin} \wedge X$, then $\pi_{D}(M T G)=\pi_{D}(k o \wedge X)$ for $D<8$; so, for the dimension $D=t-s<8$, from (E2), we have

$$
\operatorname{Ext}_{\mathcal{A}_{2}(1)}^{s, t}\left(\mathrm{H}^{*}\left(X, \mathbb{Z}_{2}\right), \mathbb{Z}_{2}\right) \Rightarrow\left(\Omega_{D=t-s}^{G}\right)_{2}^{\wedge} \text {. }
$$

Hence, we have the Adams spectral sequence,

$$
\operatorname{Ext}_{\mathcal{A}_{2}(1)}^{s, t}\left(\mathrm{H}^{*+10}\left(M \operatorname{SO}(10), \mathbb{Z}_{2}\right), \mathbb{Z}_{2}\right) \Rightarrow \Omega_{D=t-s}^{\frac{\operatorname{Spin} \times \operatorname{Spin}(10)}{\mathbb{Z}_{2}^{F}}} .
$$

Actually, we find [55]

$$
\Omega_{D}^{\frac{\operatorname{Spin} \times \operatorname{Spin}(10)}{\mathbb{Z}_{2}^{F}}}=\Omega_{D}{ }^{\frac{\operatorname{Spin} \times \operatorname{Spin}(18)}{\mathbb{Z}_{2}^{F}}}=\Omega_{D}^{\frac{\operatorname{Spin} \times \operatorname{Spin}(n)}{\mathbb{Z}_{2}^{F}}}=\Omega_{D}{ }^{\frac{\operatorname{Spin} \times \operatorname{Spin}(n+1)}{\mathbb{Z}_{2}^{F}}},
$$

for $n \geqslant 7$ and $0 \leqslant D \leqslant 6$.

\begin{tabular}{|c|c|c|}
\hline \multicolumn{3}{|r|}{ Bordism group } \\
\hline$D$ & $\Omega_{D}^{\frac{\operatorname{Spin} \times \operatorname{Spin}}{\mathbb{Z}_{2}^{f}}}$ & Generators \\
\hline 5 & $\mathbb{Z}_{2}$ & $\mathrm{w}_{2}(T M) \mathrm{w}_{3}(T M)=\mathrm{w}_{2}\left(V_{\mathrm{SO}(10)}\right) \mathrm{w}_{3}\left(V_{\mathrm{SO}(10)}\right)$ \\
\hline
\end{tabular}

TABLE III. Bordism group. The same result holds for

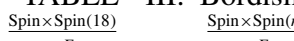
$\Omega_{D}{ }^{\frac{\mathbb{Z}_{2}^{F}}{2}}$ and $\Omega_{D}{ }^{\frac{\mathbb{Z}_{2}^{F}}{2}}$ with $n \geqslant 7$ and $0 \leqslant D \leqslant 6$. See Ref. [55]. 
TABLE IV. Topological phase classification $(\equiv \mathrm{TP})$ as a cobordism group, following Table III. Same result for $\operatorname{TP}_{D}\left(\frac{\operatorname{Spin} \times \operatorname{Spin}(18)}{\mathbb{Z}_{2}^{F}}\right)$ and $\operatorname{TP}_{D}\left(\frac{\operatorname{Spin} \times \operatorname{Spin}(n)}{\mathbb{Z}_{2}^{F}}\right)$ with $n \geqslant 7$ and $0 \leqslant D \leqslant 5$. See, also, Ref. [55].

\begin{tabular}{lcc}
\hline \hline & Cobordism group \\
\hline$D$ & $\mathrm{TP}_{D}\left(\frac{\operatorname{Spin} \times \operatorname{Spin}(10)}{\mathbb{Z}_{2}^{f}}\right)$ & Generators \\
\hline 5 & $\mathbb{Z}_{2}$ & $\mathrm{w}_{2}(T M) \mathrm{w}_{3}(T M)=\mathrm{w}_{2}\left(V_{\mathrm{SO}(10)}\right) \mathrm{w}_{3}\left(V_{\mathrm{SO}(10)}\right)$ \\
\hline \hline
\end{tabular}

The $\mathcal{A}_{2}(1)$-module structure of $\mathrm{H}^{*+10}\left(\operatorname{MSO}(10), \mathbb{Z}_{2}\right)$ below degree 6 is shown in Ref. [55], Sec. 6, Fig. 27, and the $E_{2}$ page is shown in Fig. 3. Here we have used the correspondence between the $\mathcal{A}_{2}(1)$-module structure and the $E_{2}$ page shown in Appendix A of Ref. [55].

To read the result from the Adams chart in Fig. 3, we look at the stabilized $E_{\infty}$ page, where one dot indicates a finite group $\mathbb{Z}_{p}$, and a vertical finite line segment connecting $n$ dots indicates a finite group $\mathbb{Z}_{p^{n}}$. But when $n=\infty$, the infinite line connecting infinite dots indicates an infinite group, an integer $\mathbb{Z}$. Here, $p$ is given by the $\bmod p$ Steenrod algebra $\mathcal{A}_{p}$ in (E2). Here, in Fig. 3, $p=2$, and we can read from the

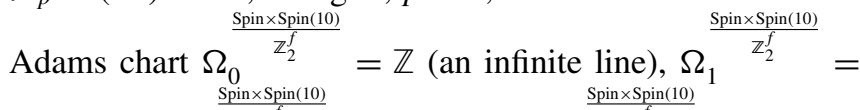
0 (nothing), $\Omega_{2}^{\frac{\frac{S \operatorname{pin} \times S \operatorname{Spin}(10)}{\mathbb{Z}_{2}^{f}}}{2}}=0$ (nothing), $\Omega_{3}^{\frac{\text { Spin } \times \operatorname{Spin}(10)}{\mathbb{Z}_{2}^{f}}}=0$ (nothing), $\Omega_{4}^{\frac{\operatorname{Spin} \times \operatorname{Sin}(10)}{\mathbb{Z}_{2}^{f}}}=\mathbb{Z}^{2}$ (two infinite lines), $\Omega_{5}^{\frac{\operatorname{Spin} \times \operatorname{Spin}(10)}{\mathbb{Z}_{2}^{f}}}=\mathbb{Z}_{2}(\mathrm{a}$ dot), and $\Omega_{6}^{\frac{\operatorname{Spin} \times \operatorname{Spin}(10)}{\mathbb{Z}_{2}^{f}}}=\mathbb{Z}_{2}$ (a dot).

\section{Classification of all invertible anomalies of $\frac{\operatorname{Spin} \times \operatorname{Spin}(10)}{\mathbb{Z}_{2}^{f}}$ and $\frac{\operatorname{Spin} \times \operatorname{Spin}(18)}{\mathbb{Z}_{2}^{f}}$ fermion theories}

By (E2) and (E3), we obtain the bordism group $\Omega_{D}^{\frac{\text { Spin } \times \text { Spin(10) }}{\mathbb{Z}_{2}^{f}}}$ shown in Table III, focusing on $D=5$.

By (E5) and (E6), we obtain the cobordism group $\mathrm{TP}_{D}\left(\frac{\operatorname{Spin} \times \operatorname{Spin}(10)}{\mathbb{Z}_{2}^{f}}\right)$ shown in Table IV, focusing on $D=5$.
[1] S. L. Glashow, Nucl. Phys. 22, 579 (1961).

[2] S. Weinberg, Phys. Rev. Lett. 19, 1264 (1967).

[3] A. Salam and J. Ward, Phys. Lett. 13, 168 (1964).

[4] D. Tong, J. High Energy Phys. 07 (2017) 104.

[5] H. Georgi and S. L. Glashow, Phys. Rev. Lett. 32, 438 (1974).

[6] H. Fritzsch and P. Minkowski, Ann. Phys. 93, 193 (1975).

[7] T. D. Lee and C. N. Yang, Phys. Rev. 104, 254 (1956).

[8] H. B. Nielsen and M. Ninomiya, Phys. Lett. B 105, 219 (1981).

[9] J. B. Kogut, Rev. Mod. Phys. 51, 659 (1979).

[10] P. H. Ginsparg and K. G. Wilson, Phys. Rev. D 25, 2649 (1982).

[11] D. B. Kaplan, Phys. Lett. B 288, 342 (1992).

[12] Y. Shamir, Nucl. Phys. B 406, 90 (1993).

[13] R. Narayanan and H. Neuberger, Phys. Lett. B 302, 62 (1993).

[14] M. Lüscher, Nucl. Phys. B 549, 295 (1999).

[15] X. Chen, Z.-X. Liu, and X.-G. Wen, Phys. Rev. B 84, 235141 (2011).

[16] X. Chen, Z.-C. Gu, Z.-X. Liu, and X.-G. Wen, Phys. Rev. B 87, 155114 (2013).

[17] J. Wang and X.-G. Wen, arXiv:1307.7480.

[18] J. Wang, X.-G. Wen, and E. Witten, Phys. Rev. X 8, 031048 (2018).

[19] E. Eichten and J. Preskill, Nucl. Phys. B 268, 179 (1986).

[20] I. Montvay, Nucl. Phys. B Proc. Suppl. 29, 159 (1992).

[21] P. D. V. Swift, Phys. Lett. B 145, 256 (1984).

[22] J. Smit, Acta Phys. Pol. B 17, 531 (1986).

[23] X.-G. Wen, Chin. Phys. Lett. 30, 111101 (2013).

[24] M. F. Golterman, D. N. Petcher, and E. Rivas, Nucl. Phys. B 395, 596 (1993).

[25] L. Lin, Phys. Lett. B 331, 449 (1994).

[26] M. F. L. Golterman and Y. Shamir, Phys. Rev. D 51, 3026 (1995).

[27] C. Chen, J. Giedt, and E. Poppitz, J. High Energy Phys. 04 (2013) 131

[28] T. Banks and A. Dabholkar, Phys. Rev. D 46, 4016 (1992).

[29] D. S. Freed and M. J. Hopkins, arXiv:1604.06527.
[30] Z. Wan and J. Wang, Ann. Math. Sci. Appl. 4, 107 (2019).

[31] Z. Wan, J. Wang, and Y. Zheng, arXiv:1912.13504.

[32] Z. Wan and J. Wang, Nucl. Phys. B (2019), doi:10.1016/j.nuclphysb.2020.115016.

[33] D. Gaiotto, A. Kapustin, N. Seiberg, and B. Willett, J. High Energy Phys. 02 (2015) 172.

[34] A. Kitaev, Ann. Phys. 303, 2 (2003).

[35] X.-G. Wen, Phys. Rev. Lett. 90, 016803 (2003).

[36] M. Levin and X.-G. Wen, Phys. Rev. B 67, 245316 (2003).

[37] B. Yoshida, Ann. Phys. 326, 15 (2011).

[38] H. Bombín, Commun. Math. Phys. 327, 387 (2014).

[39] Z. Nussinov and G. Ortiz, Proc. Natl. Acad. Sci. 106, 16944 (2009).

[40] Z. Nussinov and G. Ortiz, Ann. Phys. 324, 977 (2009).

[41] L. Kong and X.-G. Wen, arXiv:1405.5858.

[42] D. S. Freed, arXiv:1406.7278.

[43] S. Galatius, I. Madsen, U. Tillmann, and M. Weiss, Acta Math. 202, 195 (2009).

[44] R. Thom, Comment. Math. Helvetici 28, 17 (1954).

[45] X.-G. Wen, Phys. Rev. B 99, 205139 (2019).

[46] Z.-C. Gu and X.-G. Wen, Phys. Rev. B 80, 155131 (2009).

[47] T. Senthil, Ann. Rev. Condensed Matter Phys. 6, 299 (2015).

[48] A. Kapustin, arXiv:1403.1467.

[49] A. Kapustin, R. Thorngren, A. Turzillo, and Z. Wang, J. High Energy Phys. 12 (2015) 52.

[50] M. Guo, P. Putrov, and J. Wang, Ann. Phys. 394, 244 (2018).

[51] C. Wang and T. Senthil, Phys. Rev. B 89, 195124 (2014); 91, 239902(E) (2015).

[52] Q.-R. Wang and Z.-C. Gu, Phys. Rev. X 8, 011055 (2018).

[53] Q.-R. Wang and Z.-C. Gu, arXiv:1811.00536.

[54] X. Chen, Z.-C. Gu, and X.-G. Wen, Phys. Rev. B 82, 155138 (2010).

[55] Z. Wan and J. Wang, arXiv:1910.14668.

[56] A. P. Schnyder, S. Ryu, A. Furusaki, and A. W. W. Ludwig, Phys. Rev. B 78, 195125 (2008). 
[57] A. Kitaev, in Advances in Theoretical Physics: Landau Memorial Conference, Chernogolovka, Russia, 2008, AIP Conf. Proc. No. 1134, edited by V. Lebedev and M. Feigelman (AIP, Melville, NY, 2009), p. 22.

[58] A. P. Schnyder, S. Ryu, A. Furusaki, and A. W. W. Ludwig, in Advances in Theoretical Physics: Landau Memorial Conference, edited by V. Lebedev and M. Feigelman, AIP Conf. Proc. No. 1134 (AIP, New York, 2009), p. 10.

[59] Z.-C. Gu and X.-G. Wen, Phys. Rev. B 90, 115141 (2014).

[60] D. Gaiotto and A. Kapustin, Int. J. Mod. Phys. A 31, 1645044 (2016).

[61] A. Kapustin and R. Thorngren, J. High Energy Phys. 10 (2017) 080.

[62] J. Wang, K. Ohmori, P. Putrov, Y. Zheng, Z. Wan, M. Guo, H. Lin, P. Gao, and S.-T. Yau, Prog. Theor. Exp. Phys. 2018, 053A01 (2018).

[63] M. Guo, K. Ohmori, P. Putrov, Z. Wan, and J. Wang, Commun. Math. Phys. 376, 1073 (2020).

[64] T. Lan, C. Zhu, and X.-G. Wen, Phys. Rev. B 100, 235141 (2019)..

[65] Z. Wan, J. Wang, and Y. Zheng, Ann. Phys. 414, 168074 (2020).

[66] I. García-Etxebarria and M. Montero, J. High Energy Phys. 08 (2019) 003.

[67] J. Wang, X.-G. Wen, and E. Witten, J. Math. Phys. 60, 052301 (2019).

[68] J. Milnor and J. Stasheff, Characteristic Classes, by Milnor and Stasheff, Annals of Mathematics Studies, Vol. 76 (Princeton University Press, Princeton, NJ, 1974).

[69] C. Arf, J. Reine Angew. Math. 183, 148 (1941).

[70] A. Y. Kitaev, Phys. Usp. 44, 131 (2001).

[71] M. DeMarco and X.-G. Wen, arXiv:1706.04648.

[72] Y. BenTov and A. Zee, Phys. Rev. D 93, 065036 (2016).

[73] Y.-Z. You, Y. BenTov, and C. Xu, arXiv:1402.4151.

[74] Y.-Z. You and C. Xu, Phys. Rev. B 91, 125147 (2015).

[75] Y. Kikukawa, Prog. Theor. Exp. Phys. 2019, $073 B 02$ (2019).

[76] Y. Kikukawa, Prog. Theor. Exp. Phys. 2019, 113B03 (2019).

[77] J. Wang and X.-G. Wen, Phys. Rev. D 99, 111501(R) (2019).

[78] G. Baskaran and P. W. Anderson, Phys. Rev. B 37, 580 (1988).

[79] I. Affleck, Z. Zou, T. Hsu, and P. W. Anderson, Phys. Rev. B 38, 745 (1988).
[80] E. Dagotto, E. Fradkin, and A. Moreo, Phys. Rev. B 38, 2926 (1988).

[81] R. C. Ball, Phys. Rev. Lett. 95, 176407 (2005).

[82] F. Wilczek and A. Zee, Phys. Rev. D 25, 553 (1982).

[83] X.-G. Wen, Phys. Rev. Lett. 88, 011602 (2001).

[84] X.-G. Wen, Phys. Rev. D 68, 065003 (2003).

[85] M. Levin and X.-G. Wen, Phys. Rev. B 73, 035122 (2006).

[86] J. A. Wheeler, Information, physics, quantum: The search for links, in Feynman and Computation: Exploring the Limits of Computers (Perseus Books, USA, 1989), pp. 309-336.

[87] M. A. Levin and X.-G. Wen, Phys. Rev. B 71, 045110 (2005).

[88] X.-G. Wen, Condens. Matter Phys. 20, 198710 (2013).

[89] G. 't Hooft, NATO Adv. Study Inst. Ser. B Phys. 59, 135 (1980).

[90] X.-G. Wen, Phys. Rev. D 88, 045013 (2013).

[91] C. Callan and J. Harvey, Nucl. Phys. B 250, 427 (1985).

[92] D. S. Freed, J. Differential Geom. 80, 45 (2008).

[93] E. Witten, Commun. Math. Phys. 100, 197 (1985).

[94] X. Dai and D. S. Freed, J. Math. Phys. 35, 5155 (1994).

[95] L. Kong, X.-G. Wen, and H. Zheng, arXiv:1502.01690.

[96] D. Fiorenza and A. Valentino, Commun. Math. Phys. 338, 1043 (2015).

[97] S. Monnier, Commun. Math. Phys. 338, 1327 (2015).

[98] W. Ji and X.-G. Wen, Phys. Rev. Res. 1, 033054 (2019).

[99] J. C. Wang, L. H. Santos, and X.-G. Wen, Phys. Rev. B 91, 195134 (2015).

[100] A. Kapustin and R. Thorngren, arXiv:1404.3230.

[101] E. Witten, Phys. Lett. B 117, 324 (1982).

[102] X. G. Wen, Int. J. Mod. Phys. B 04, 239 (1990).

[103] A. Kapustin, arXiv:1404.6659.

[104] J. C. Wang, Z.-C. Gu, and X.-G. Wen, Phys. Rev. Lett. 114, 031601 (2015).

[105] A. Vishwanath and T. Senthil, Phys. Rev. X 3, 011016 (2013).

[106] S. L. Adler, Phys. Rev. 177, 2426 (1969).

[107] J. Bell and R. Jackiw, Nuovo Cimento A 60, 47 (1969).

[108] J. C. Wang and X.-G. Wen, Phys. Rev. B 91, 125124 (2015).

[109] A. Kapustin, Phys. Rev. B 89, 125307 (2014).

[110] A. Kitaev and L. Kong, Commun. Math. Phys. 313, 351 (2012).

[111] T. Lan, J. C. Wang, and X.-G. Wen, Phys. Rev. Lett. 114, 076402 (2015).

[112] N. Seiberg (unpublished). 